\title{
Ground target recognition using rectangle
} estimation

\author{
Christina Grönwall, Fredrik Gustafsson, Mille Millnert \\ Division of Automatic Control \\ Department of Electrical Engineering \\ Linköpings universitet, SE-581 83 Linköping, Sweden \\ WWW: http://www.control.isy.liu.se \\ E-mail: stina@isy.liu.se, fredrik@isy.liu.se, \\ mille@isy.liu.se
}

2nd June 2006

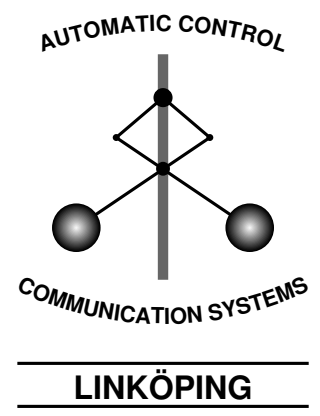

Report no.: LiTH-ISY-R-2735

Accepted for publication in IEEE Transactions on Image Processing

Technical reports from the Control \& Communication group in Linköping are available at http://www.control.isy.liu.se/publications 


\begin{abstract}
We propose a ground target recognition method based on 3D laser radar data. The method handles general 3D scattered data. It is based on the fact that manmade objects of complex shape can be decomposed to a set of rectangles. The ground target recognition method consists of four steps; 3D size and orientation estimation, target segmentation into parts of approximately rectangular shape, identification of segments that represent the targets functional/main parts and target matching with CAD models. The core in this approach is rectangle estimation. The performance of the rectangle estimation method is evaluated statistically using Monte Carlo simulations. A case study on tank recognition is shown, where 3D data from four fundamentally different types of laser radar systems are used. Although the approach is tested on rather few examples, we believe that the approach is promising.
\end{abstract}

Keywords: Rectangle estimation, laser radar, automatic target recognition 


\title{
Ground target recognition using rectangle estimation
}

\author{
Christina Grönwall, Fredrik Gustafsson, Mille Millnert
}

\begin{abstract}
We propose a ground target recognition method based on 3D laser radar data. The method handles general 3D scattered data. It is based on the fact that man-made objects of complex shape can be decomposed to a set of rectangles. The ground target recognition method consists of four steps; 3D size and orientation estimation, target segmentation into parts of approximately rectangular shape, identification of segments that represent the target's functional/main parts and target matching with CAD models.

The core in this approach is rectangle estimation. The performance of the rectangle estimation method is evaluated statistically using Monte Carlo simulations. A case study on tank recognition is shown, where 3D data from four fundamentally different types of laser radar systems are used. Although the approach is tested on rather few examples, we believe that the approach is promising.
\end{abstract}

Index Terms - Rectangle estimation, laser radar, automatic target recognition

\section{INTRODUCTION}

\section{A. Ground target recognition using 3D imaging laser radar}

Laser radar systems have been investigated over several decades primarily for military applications [19, 25, 26]. The high resolution in angle-angle-range makes $3 \mathrm{D}$ imaging possible and due to the short wavelength, in general $0.5-10$ $\mu m$, detailed range images of objects and background can be obtained. Due to the high resolution, even at $\mathrm{km}$ distances, details of a target can be resolved. This can be used for automatic target recognition (ATR). If different functional parts of a vehicle can be identified or a building of complex shape can be decomposed into pieces that are easier to process, the recognition task can be simplified. If a factory's chimney or the driver's compartment of a truck can be identified we have an indication of the object type or identity besides the model matching. When performing matching, the list of possible models has been reduced. Further, if the object's articulated parts can be identified, the recognition is simplified as the degrees of freedom reduce.

In this paper we propose a ground target recognition method based on 3D laser radar data. The method handles general 3D scattered data. It is based on the fact that man-made objects of complex shape can be decomposed into a set of rectangles. The method consists of four steps; 1) estimation of the target's 3D size and orientation, 2) segmentation of the target into parts of approximately rectangular shape, 3) identification of segments that contain the functional (main) parts of the target and 4) matching the target with library models.

C. Grönwall is with the Swedish Defence Research Agency, Dept. of Laser Systems, Linköping, Sweden. E-mail: christina.gronwall@foi.se. Her former surname was Carlsson.

F. Gustafsson and M. Millnert are with the Dept. of Electrical Engineering, Linköping University, Linköping, Sweden. E-mail: \{fredrik,mille\}@isy.liu.se
From a computer vision perspective, this sequential processing of data is not optimal. An advantage is that even if a matching model cannot be found, we can report the estimated size and orientation and possibly some identified features.

\section{B. The ATR framework}

The framework of the target recognition method proposed in this paper is described in $[3,4,15]$. The framework is a query-based multi sensor information system for ground target recognition. Based on an operator's query, the system selects proper sensor data and analysis algorithms to perform the task. Once the target is detected, a four-step target recognition process is performed. The recognition is based on infrared, visual and laser radar data. First the sensor data is analyzed to estimate target attributes, for example position, dimensions and temperature. The attributes from different algorithms are then fused. Based on the attribute fusion, models of typical military vehicles are selected and the models are matched with sensor data. The model library contains wire-frame CAD models with thermal and visual textures. The results from the model matching are then subject to model match fusion and finally, the most likely match results are presented to the operator. The method described in this paper is used both in the attribute estimation and in the model matching.

\section{Outline}

In the next section, we review some of the ATR work based on laser radar data and methods for rectangle estimation. In Section III, the rectangle estimation method is described and analyzed. In Section IV, the segmentation of objects with complex shape is described. In Section V, we propose a ground target recognition method based on rectangle estimation and in Section VI it is applied to tank recognition. The results and future work are discussed in Section VII and in Section VIII we conclude this paper.

\section{RELATED WORK}

\section{A. Vehicle recognition using laser radar}

Several ATR methods or systems for recognition of military ground vehicles based on laser radar data have been proposed over the years $[4,10,30,31,33,35]$. During the last years, also ATR of civilian personal cars, mainly for traffic monitoring, have been proposed [16, 28, 34].

The approaches are applied to data of different resolution and different perspectives of the target. In [4, 28, 33]-[35], low resolution data is considered. A typical data set contains up to a few hundreds samples on a target, while [28] handles very low-resolution data (approx. 1.5 points $/ \mathrm{m}^{2}$ ). In $[10,16$, $30,31]$, there are typically several hundreds of samples on 
a target. Typically, the data is collected in a forward-looking perspective, while in [4] and [28] down-looking perspective data is considered. Often data is obtained using a scanning laser radar system, which results in irregularly sampled data. In $[16,33]$, both simulated and real data are used. In [30, $31,35]$, the laser sensor works in staring mode, which gives regularly sampled data. Further, in [16, 30], data is collected from several views, which results in data that is less selfoccluding.

In most cases, the ATR process is divided into two steps. Usually the first step consists of fast feature extraction or silhouette calculations $[10,31,33,34]$. The feature extraction can retrieve geometrical properties of the target [4], lowerdimensional properties [35] or more abstract features like spin image representation [16, 30] (see [20] for description of spin images). The first step is used to reduce the list of potential targets. Then, the remaining targets are subject to $3 \mathrm{D}$ matching with library models, which are represented by CAD models $[4,10]$, some representation generated from CAD models [31, $33,35]$ or $3 \mathrm{D}$ scatter data $[10,30,34]$. The ATR approach [35] is further evaluated in [18]. In [28], learning is used for the recognition. The methods in $[10,16,30]$ can handle partly occluded targets. The problem with partly occluded targets is discussed, for example, in [4].

\section{B. Rectangle estimation for complex shape analysis}

When analyzing an object with complex shape, registered in $2 \mathrm{D}$ by passive imaging or projection of $3 \mathrm{D}$ data, the orientation can be estimated by rectangle fitting. An iterative approach is proposed in [12]. In [9, 32, 36], non-iterative approaches to rectangle estimation are used to find good initial values for further processing. The objects that are characterized are asteroids [36], buildings [32] and vehicles [9], respectively. In $[32,36]$, eigenvalue calculations are used to estimate the orientation of the object. After that, a rectangle that bounds the object samples [36] or is optimal in second order moment [32] is calculated. In [9], a rectangle that bounds the object data is estimated by solving an optimization problem, which is described further in Section III.

\section{RECTANGLE ESTIMATION}

\section{A. Definition}

The current approach for rectangle estimation has been described independently under the name Rotating Calipers [29] and in [8,9]. This rectangle estimation approach is more general than the methods based on principal axis estimation $[32,36]$, as there is no demand that the orientation scatter matrix must be positive definite.

We describe the rectangle estimation problem as an optimization problem. A straight line in two dimensions is described as $n_{1} x+n_{2} y-c=0$, where the normal vector $\mathbf{n}=\left(n_{1}, n_{2}\right)^{T}$ defines the slope of the line and $c$ the distance to origin and $(x, y)$ is measurement data known to be on the object, possibly contaminated with noise. The points $\left(x_{1}, y_{1}\right),\left(x_{2}, y_{2}\right), \ldots,\left(x_{N}, y_{N}\right)$ are inside the rectangle or on

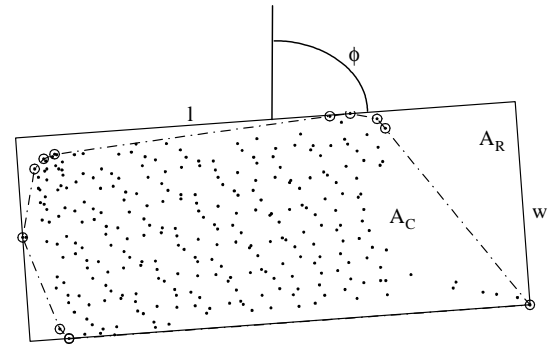

Fig. 1. Illustration of the parameters estimated in the rectangle estimation. A set of samples (dots), the convex hull (dashed line) and the estimated rectangle (solid line). The samples belonging to the convex hull are encircled. The parameters are length $(1)$, width $(\mathrm{w})$, orientation $(\phi)$, convex hull area $\left(A_{C}\right)$ and rectangle area $\left(A_{R}\right)$.

one of the sides of the rectangle if

$$
\begin{aligned}
& \text { Side } 1: n_{1} x_{i}+n_{2} y_{i}-c_{1} \geq 0, \quad i=1, \ldots, N \\
& \text { Side } 2: \quad-n_{2} x_{i}+n_{1} y_{i}-c_{2} \geq 0, \quad i=1, \ldots, N \\
& \text { Side } 3: n_{1} x_{i}+n_{2} y_{i}-c_{3} \leq 0, \quad i=1, \ldots, N \\
& \text { Side } 4: \quad-n_{2} x_{i}+n_{1} y_{i}-c_{4} \leq 0, \quad i=1, \ldots, N,
\end{aligned}
$$

where $\mathbf{n}^{T} \mathbf{n}=1$. If we introduce $X_{i}=\left(x_{i}, y_{i}\right)$ and the rotation matrix

$$
R^{+}=\left(\begin{array}{cc}
0 & -1 \\
1 & 0
\end{array}\right),
$$

we can formulate the rectangle estimation problem as a minimization problem, where the rectangle's area is the objective function:

$$
\min \left(c_{3}-c_{1}\right)\left(c_{4}-c_{2}\right)
$$

subject to

$$
\begin{aligned}
& X_{1, i} \mathbf{n}-c_{1} \geq 0, \quad i=1, \ldots, N \\
& X_{1, i} R^{+} \mathbf{n}-c_{2} \geq 0, \quad i=1, \ldots, N \\
& X_{1, i} \mathbf{n}-c_{3} \leq 0, \quad i=1, \ldots, N \\
& X_{1, i} R^{+} \mathbf{n}-c_{4} \leq 0, \quad i=1, \ldots, N \\
& \mathbf{n}^{T} \mathbf{n}=1 .
\end{aligned}
$$

Based on the estimates of $\mathbf{n}$ and $c_{j}, j=1, \ldots, 4$, the rectangle's length, $l$, width, $w$, area, $A_{R}$, and orientation, $\phi$, are calculated, as illustrated in Figure 1.

Problem (2) is not convex, as the objective function and the last constraint are not convex, but it is proven in $[9,23]$ that there exists a unique solution. There is a constraint that limits the number of possible orientations of the rectangle, see Theorem 1.

Theorem 1 (Minimal rectangle): The rectangle of minimum area enclosing a convex polygon has a side colinear with one of the edges of the polygon.

Proof: See [11]. The proof is also performed in $[8,9$, 23].

Using this theorem, we can limit the number of possible orientations of the rectangle, only rectangles that have one side colinear with one of the edges of the convex hull (that is a convex polygon) have to be tested.

In [9] and [29] (almost similar) algorithms are given for calculation of (2) in linear time, i.e., $O\left(N_{v}\right)$ where $N_{v}$ is 
the number of vertices in the convex polygon. Further, the convex hull can be calculated in $O(N \log N)$ time if data is unsorted and in $O(N)$ time if data is sorted ( $N$ is the number of samples). In [8] a sorting algorithm for scanned laser radar data is proposed, whose execution time is linear in the number of samples. The implementation [9], used in this paper, is based on that four samples shall span the rectangle, one sample for each side, i.e., we have $N_{v} \geq 4$.

\section{B. Performance}

Analytical derivations show that the length, width and area estimates contain bias [14]. It is shown that $\operatorname{bias}(l)=$ $-2 l_{0} /(N+1), \operatorname{bias}(w)=-2 w_{0} /(N+1)$ and $\operatorname{bias}\left(A_{R}\right)=$ $-4 N A_{0} /(N+1)^{2}$. The orientation estimate is unbiased.

The performance of the estimation method (2) is investigated in Monte Carlo simulations. The performance is evaluated in terms of correctness in estimates of $\theta=\left(l, w, \phi, A_{R}\right)$. Further, the ratio between the convex hull's area and the rectangle's area, $A_{C} / A_{R}$, is studied. We start with random placement of $N$ samples in $(x, y)$, where $x \in \mathcal{U}\left(-l^{0} / 2, l^{0} / 2\right)$ and $y \in \mathcal{U}\left(-w^{0} / 2, w^{0} / 2\right)$, respectively, where $\mathcal{U}(\cdot)$ is the uniform distribution. These samples are considered noise free. Random errors, Gaussian distributed with zero mean and equal variance $\sigma_{e_{x}}^{2}=\sigma_{e_{y}}^{2}$ are added to $(x, y)_{i}, i=1, . ., N$. The noise is generated separately for $x$ and $y$. The parameters $\theta$ are estimated using (2) on the perturbed data set. The statistical properties of the estimates are studied by the mean squared error (MSE) and bias, which are averaged over 100 sets. The MSE and the bias for parameter $\theta_{j}$ are defined as

$$
\begin{aligned}
\operatorname{MSE}\left(\hat{\theta}_{j}\right) & =E\left(\hat{\theta}_{j}-\theta_{j}^{0}\right)^{2}+E^{2}\left(\hat{\theta}_{j}-\theta_{j}^{0}\right) \\
& =\operatorname{Var}\left(\hat{\theta}_{j}\right)+\operatorname{bias}^{2}\left(\hat{\theta}_{j}\right),
\end{aligned}
$$

where $\theta_{j}^{0}$ is the true, but unknown, parameter and $\hat{\theta}_{j}$ is the estimate. The properties of the area ratio $A_{C} / A_{R}$ is studied using mean and standard deviation. The properties of the estimates are studied as a function of the number of samples, $N$, and signal to noise ratio (SNR). SNR is defined as

$$
\mathrm{SNR}=\min \left(\frac{r(x)}{\sigma_{e_{x}}}, \frac{r(y)}{\sigma_{e_{y}}}\right),
$$

where $r(x)$ is the range in data, $r(x)=x_{\max }-x_{\min }$.

The simulation results agree with the analytical expressions [14]. The results of the area ratio

$$
M=A_{C} / A_{R}
$$

are described in more detail, as it will be used in the proposed ATR process. For the area ratio $A_{C} / A_{R}$, the mean and standard deviation are studied, see Figure 2 for results of mean value simulation. For noise free data we have $1 / 2 \leq$ $A_{C} / A_{R} \leq 1$, where the lower limit is reached for three samples $(N=3)$. The upper limit is reached when there is an infinite number of samples. For a low SNR and a large number of samples the shape of convex hull will approach an ellipse, i.e., $A_{C} / A_{R} \rightarrow \frac{\pi}{4}\left(\approx 10^{-0.1}\right)$. For small samples sets, both high and low SNR, the standard deviation of the estimate is approximately $10 \%$ and when the number of samples increases the standard deviation decreases to $2-4 \%$ [14].

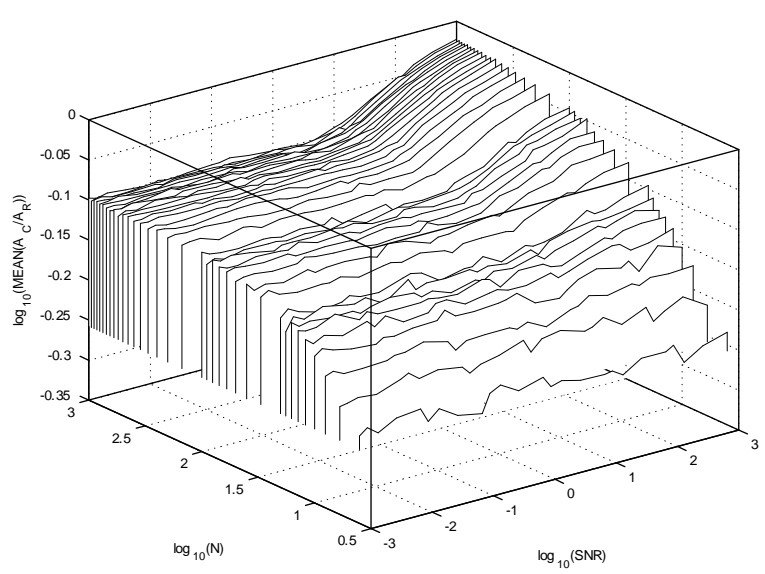

Fig. 2. Mean of $M=A_{C} / A_{R}$, as a function of number of samples $N$ and SNR.

\section{Segmentation of COMPlex Shapes}

Man-made objects, like vehicles and buildings, are in certain projections of rectangular shape. When the objects are of more complex shape, they can usually be decomposed into a set of rectangles. In this section, we describe an approach to decompose a complex shape to a set of rectangles. The approach has similarities with [32], a main difference is that it handles irregularly sampled data.

This method works on 2D data retrieved from projections of $3 \mathrm{D}$ data. If the current data set is not approximately similar to a rectangle, the data set is considered to describe a complex shape and it will be subject to segmentation. We split the object recursively by sliding a splitting line that is parallel to first the primary and then to the secondary axis of the rectangle. The data set will be traversed a certain distance $\delta$ in each iteration. Tests have shown that $\delta$ should be of the same magnitude as the searched subparts of the object. The two subsets of the object (part) that have the smallest total area are selected for segmentation. The result of the segmentation is stored in a binary tree $T$. In a tree, each terminating node (leaf), $t$, contains indices to either a rectangle-like part of the object or a part that cannot be further split.

An indication that node $t$ needs further splitting is the dissimilarity of the bounding rectangle's area and the area of the convex hull of the samples stored in node $t$. The area ratio is similar to the Hausdorff measure used in [32]. Let $A_{R}(t)$ denote the bounding rectangle's area for the samples in node $t$ and $A_{C}(t)$ the area of the convex hull for the samples in node $t$. The area ratio for $t$ is defined as

$$
M(t)=\frac{A_{C}(t)}{A_{R}(t)}
$$

where $0<M(t) \leq 1$. If $M(t)$ is smaller than a threshold $\tau$, the data set stored in node $t$ is considered not being of rectangular shape. Thus, the contents in $t$ will be split to $t_{L}$ and $t_{R}$ (i.e., left and right leaf in the binary tree). The segmentation algorithm can be summarized in six steps:

1) Calculate $M(t)$, see (5). 


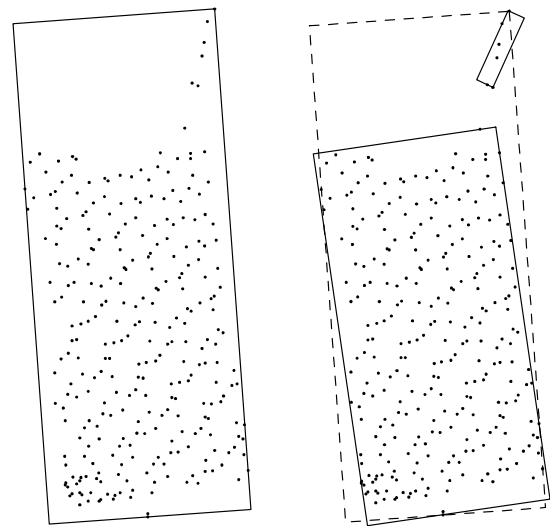

Fig. 3. Example of splitting of node $t$. Left: node $t, M(t)=0.79$. Right after splitting, the dashed rectangle is the rectangle for node $t$. Upper node is $t_{L}, M\left(t_{L}\right)=0.27$, and lower node is $t_{R}, M\left(t_{R}\right)=0.94$.

2) Calculate SNR (4) and select $\tau$ from a table.

3) If $M(t)<\tau$, proceed below. Otherwise, terminate.

4) Split node $t$ into $t_{L}$ and $t_{R}$. Do one separation for each increment $\delta$.

5) Select the $t_{L}$ and $t_{R}$ that have the smallest total rectangle area.

6) Check area ratios $M\left(t_{L}\right)$ and $M\left(t_{R}\right)$ :

a) if $M\left(t_{L}\right) \geq \tau$ or $M\left(t_{R}\right) \geq \tau$, save $t_{L}$ or $t_{R}$ respectively, and terminate.

b) if $M\left(t_{L}\right)<\tau$ or $M\left(t_{R}\right)<\tau$, segment $t_{L}$ or $t_{R}$, respectively, further.

The threshold $\tau$ is based on statistics of the number of samples and SNR (4) in the current data set. The mean of $M$ is stored in a table and for every test the proper value of $\tau$ is selected, based on the number of samples in the current node and the SNR (see Figure 2, Section III). Thus, $\tau$ is set to the value of $M$ that is expected for the current $N$ and SNR. The noise variances $\sigma_{e_{x}}^{2}$ and $\sigma_{e_{y}}^{2}$ are given by the measurement system model.

Segmentation is only performed if $N \geq 8$. An example of segmenting is shown in Figure 3. On rare occasions the samples are distributed such that the convex hull contains less than four edges. Then the bounding rectangle cannot be calculated. The bounding rectangle for the contents in node $t_{L}\left(\right.$ or $\left.t_{R}\right)$ will then be approximated by its upper bound:

$$
A_{R}\left(t_{L}\right) \leq A_{R}(t)-A_{R}\left(t_{R}\right)
$$

and the orientation will be estimated using principal component analysis.

\section{APPliCATION TO GROUND TARGET RECOGNITION}

\section{A. Introduction}

In this section, we apply the rectangle estimation and segmentation approach to recognition of man-made ground targets. The main steps of the method are described here and are illustrated in the next section.

We assume that a ground target viewed in different projections can be approximated by a set of rectangles and that in some views the rectangles will describe the functional parts of the target. When a target is measured with a laser radar, we can derive a 3D view of the object. This means that data can be projected to an arbitrary view. On the other hand, a laser beam does not penetrate dense materials like metal surfaces. Thus, we only collect data from the parts of the object that are visible from the laser radar's perspective (so-called selfocclusion). Further, in this application we cannot assume that the target is placed in a certain pose relative to the sensor and we cannot assume any certain orientation (or articulation) of the target.

The object recognition algorithm consists of four steps:

1) Estimate the target's $3 \mathrm{D}$ size and orientation using the rectangle estimation method described in Section III.

2) Segment the target into parts of approximately rectangular shape using the method described in Section IV. The main parts of the object are stored in (some of) the terminating leaves.

3) Traverse the terminating leaves and search for possible target parts by simple geometric comparisons.

4) Match the entire object with a wire-frame model. The model's functional parts are rotated to the estimated orientations.

\section{B. $3 D$ size and orientation estimation}

We first study the object in top view and then rotate to side and front/back views. The 3D size and orientation estimation consists of five steps:

1. Transform data to top view perspective.

2. Estimate a rectangle based on top view data $(x, y)$ using (2). The main directions of the target are given by the orientation of the rectangle. The yaw angle is given by the orientation of the rectangle's main axis.

3. Project the data set into the direction $\left(x^{\prime}, y^{\prime}\right)$, where $x^{\prime}$ is parallel to the main and $y^{\prime}$ is parallel to the secondary axis.

4. Estimate a rectangle based on side view data $\left(x^{\prime}, z\right)$. The pitch angle is given by the orientation of this rectangle.

5. Estimate a rectangle based on back/front view data $\left(y^{\prime}, z\right)$. The roll angle is given by this rectangle's orientation.

\section{Target segmentation and node classification}

We now have an estimate of the target's size and orientation and could continue directly with model matching. However, we will first analyze the target further by segmenting it into rectangle-like parts, in order to determine its functional parts. This is used to reduce the number of target classes in the matching step and to improve the initial fit of the target samples to the model. If articulated parts are detected the model is articulated according to the estimates and the fitting can be improved. Further, if the significant parts are identified we have information that complements the match result.

The target is segmented in top, side and back/front views and for every view both in horizontal and vertical directions. This results in six descriptions of the target, stored in six binary trees $T_{1}, \ldots, T_{6}$. In each tree the root contains indices 
to all target samples in the scene and the leaves contain indices to either a rectangle-like part or a segment that cannot be further split. We now have six different segmentations of the target, where some leaves contains the target's functional parts. To determine the typical functional parts for a target type, its model is analyzed. The model is in this case a 3D wire-frame model. The wire-frame models come from CADmodels or earlier measurements. The dimensions and allowed orientations of the functional parts and the distances between each part and the complete target are stored. The leaves of the six trees are traversed and tested against the geometric rules of the model. Leaves that apply to the rules of one or more functional parts are marked and used to orientate the model in the matching step.

\section{Matching}

The 3D data of the target will be matched with lowresolution $\mathrm{CAD}$ models (face/wire-frame models). If the target's functional parts have been identified the model's parts are rotated to the estimated orientations. Otherwise, the target will be matched with the model in default orientation.

The matching score is calculated using the relative mean squared error (RE) [7]. Let $(x, y, z)_{i}$ define target sample $i$ and $\left(x^{\prime}, y^{\prime}, z^{\prime}\right)_{i}$ the projection on the closest model facet of model $\mathcal{M}$, i.e.,

$$
\left(x^{\prime}, y^{\prime}, z^{\prime}\right)_{i}=\operatorname{Proj}\left((x, y, z)_{i} \mid \mathcal{M}\right)
$$

The RE is defined as

$$
R E=\frac{H\left((x, y, z),\left(x^{\prime}, y^{\prime}, z^{\prime}\right)\right)}{S(x, y, z)},
$$

where $H\left(\left(x^{\prime}, y^{\prime}, z^{\prime}\right),(x, y, z)\right)$ is the Euclidian distance

$H\left((x, y, z),\left(x^{\prime}, y^{\prime}, z^{\prime}\right)\right)=\frac{1}{2 N} \sum_{i=1}^{N}\left\|(x, y, z)_{i}-\left(x^{\prime}, y^{\prime}, z^{\prime}\right)_{i}\right\|_{2}^{2}$,

and $S(x, y, z)$ is the spread in data estimated by

$$
S(x, y, z)=\frac{1}{N} \sum_{i=1}^{N}\left\|(x, y, z)_{i}-\bar{\mu}\right\|_{2}^{2},
$$

where $\bar{\mu}=\left(\bar{\mu}_{x}, \bar{\mu}_{y}, \bar{\mu}_{z}\right)$ is the estimated mean value. The RE is always nonnegative and for good initial fits of model and target we have $H\left((x, y, z),\left(x^{\prime}, y^{\prime}, z^{\prime}\right)\right)<S(x, y, z)$ [7], thus $0 \leq R E<1$.

The matching score can be improved by least squares fitting [6]. In this approach we minimize the distance between the target's samples and their projected samples, i.e.,

$$
\min _{R, T} \sum_{i=1}^{N}\left\|(x, y, z)_{i}-\left(\left(x^{\prime}, y^{\prime}, z^{\prime}\right)_{i} R+T\right)\right\|,
$$

where $R$ is the rotation matrix and $T$ the translation.

\begin{tabular}{|l|l|l|l|}
\hline System & Tanks & APC & AAG \\
\hline Down-looking & $5 \mathrm{~T} 72$ & 2 BTR70 & \\
\hline Side-looking & $4 \mathrm{~T} 72$ & 6 MTLB & \\
\hline Side-looking, GV & $1 \mathrm{~T} 72$ & & \\
\hline Forward-looking & $12 \mathrm{M} 60$ & $2 \mathrm{M} 113$ & 1 ZSU23 \\
\hline
\end{tabular}

TABLE I

THE NUMBER OF TARGETS REGISTERED WITH EACH SYSTEM.

\section{CASE STUdy: TANK RECOGNITION}

In this section the steps of a target recognition process are demonstrated on tank recognition. The examples show registrations of tanks, armored personal carriers (APC) and an anti-aircraft gun (AAG) performed with four fundamentally different types of laser radar systems. Three of the laser radars register both $3 \mathrm{D}$ and reflectance, but the reflectance data is not used in this paper. Most targets are placed in open terrain with no occluding objects, but some are placed in forested areas.

\section{A. The data sets}

In this test we have two types of tanks, three types of APC and one AAG originating from four different laser radar systems, see Table I. The target models are shown in Figure 4. One set of data comes from a helicopter-borne down-looking scanning laser $\operatorname{radar}^{1}$ [17]. The measurement model of this system is given in [13]. Typically, there are 70-250 samples on a target. There are registrations performed with a sidelooking, scanning laser radar system ${ }^{2}$. Typically, there are $700-$ 1300 samples on a target. From another side-looking system we have a registration of a T72 tank (1300 samples). This system is scanning in range, a so-called gated viewing system [24]. Using [5], the set of 2D intensity images is transformed into a regular grid with range information (3D data of the scene). Finally, there is data from a forward-looking system with aspect angle 60-70 degrees. Typically, there are 200-400 samples on a target. Due to reflectance properties of cylinderlike surfaces and the measurement situations, 4 tanks (1 T72 and $3 \mathrm{M} 60$ ) do not have any registrations on their barrels.

\section{B. Preprocessing}

We assume that the target scene is detected $[3,4,15]$. A scene of approximately $15 \times 15$ meters containing the target is selected manually. When the targets are placed in open terrain, we apply the 3D size and orientation estimation algorithm (Section V-B) on all scene data to estimate the ground's slope. We rotate the scene data so that the ground is approximately a flat surface. The ground and target samples are then separated using height differentiation. For targets placed in forested areas the target samples are selected manually.

\section{C. $3 D$ size and orientation estimation}

The 3D orientation and size estimates are illustrated in Figures 8-10. In Figures 5-7 the results on tanks and MTLB are shown. For the tanks the estimations including turret are

\footnotetext{
${ }^{1}$ The TopEye system from TopEye AB, see www.topeye.com.

${ }^{2}$ The 3D-ILRIS system from OpTech Inc., see www.optech.on.ca.
} 

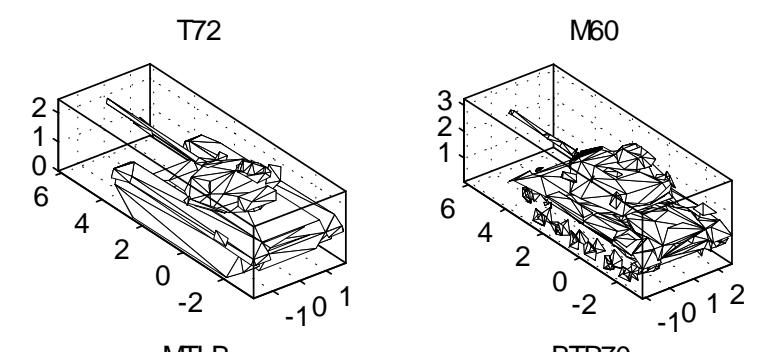

MTLB

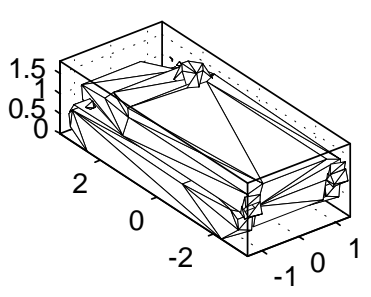

M113
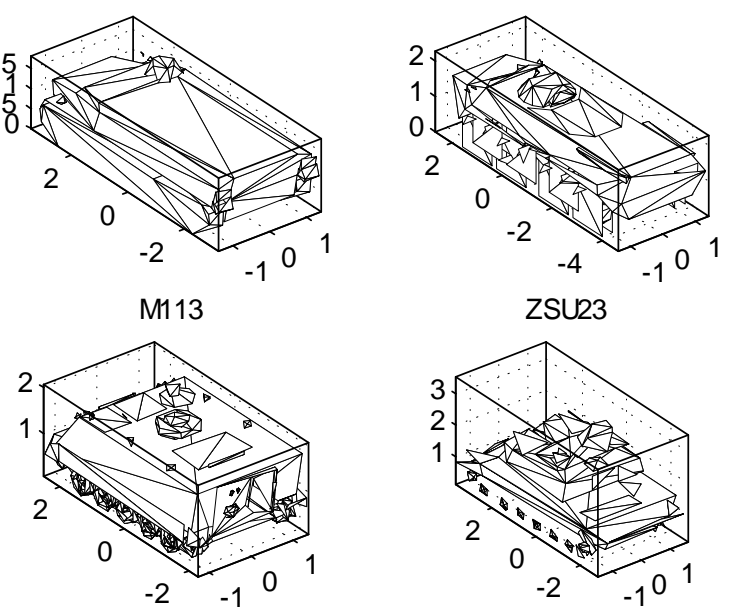

ZSU23

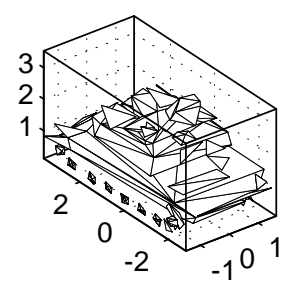

Fig. 4. Models of the targets.

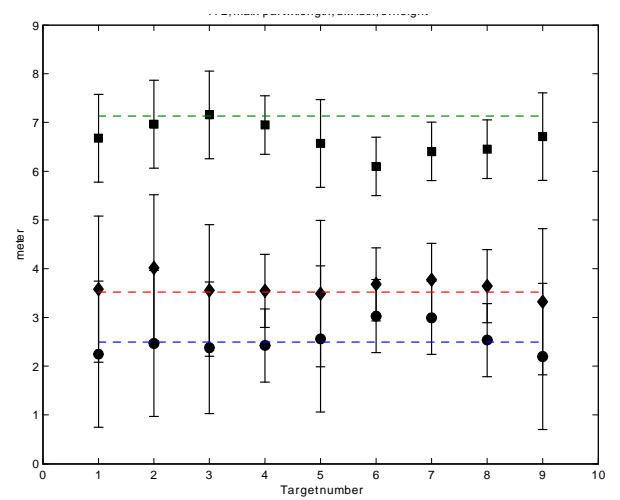

Fig. 5. Dimension estimates for T72 registrations, barrel samples excluded. The lines shows true values of length (top), width (middle) and height (bottom). Estimates length (squares), width (diamonds), height (circles). The $3 \sigma$ limit is shown, bias excluded.

shown, identified barrel samples are excluded. Using a three standard deviation limit, the width and height estimates are close to the true values. The true values come from the model library. The length estimates are underestimated, even with the three standard deviation limit and bias included. The length measurements give large range values, which give high SNR values and small MSE values, and the standard deviation follows. The length value can also be underestimated due to the measurement techniques, with side- and forwardlooking perspective, where the whole target may not always be registered, see example in Figure 10. The results shown in Figures 5-7 are representative for tanks with barrel included, the other APC:s and the AAG.

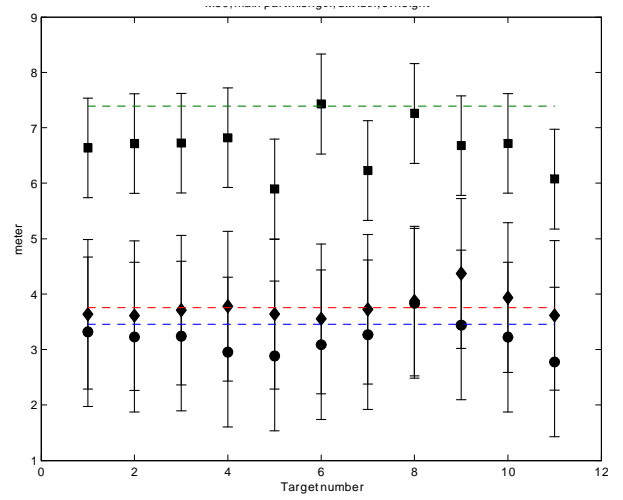

Fig. 6. Dimension estimates for M60 registrations, barrel samples excluded. The lines shows true values of length (top), width (middle) and height (bottom). Estimates length (squares), width (diamonds), height (circles). The $3 \sigma$ limit is shown, bias excluded.

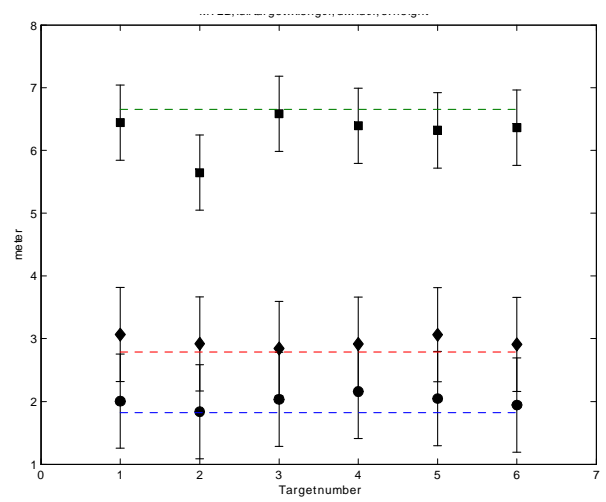

Fig. 7. Dimension estimates for MTLB registrations. The lines shows true values of length (top), width (middle) and height (bottom). Estimates length (squares), width (diamonds), height (circles). The $3 \sigma$ limit is shown, bias excluded.

\section{Target segmentation and node classification}

In this case we set $\delta=0.5$ meter to detect the functional parts barrel and turret of a tank. The geometric rules for barrel identification are: $1 \mathrm{~m}$ (meter) above ground level, maximum width $0.7 \mathrm{~m}$, length $0.7-3 \mathrm{~m}$, max. distance from target's inertia $3 \mathrm{~m}$, barrel yaw $-\pi / 3-\pi / 3$ and barrel pitch $-\pi / 18-\pi / 3$ relative to target. The geometric rules for turret identification are: $1 \mathrm{~m}$ above ground level, length/width between 1.5-5 m, height between .25-3 m, max. distance from target's inertia $1 \mathrm{~m}$, turret pitch $-\pi / 18-\pi / 18$ relative to target and the maximum area in top view is max. $50 \%$ of the whole target.

Figure 8 shows the segmentations of a T72 target, registered in down-looking perspective. For this target the functional parts of a tank were identified in the side view projection. In Figures 9-10 segmentation results for the side-looking systems are shown. In Table II the results for all targets are shown. For one tank the two barrels' samples are connected to chassis samples and not identified. In one case both the correct barrel and a false one are detected. For half of the tank examples there are only 1-5 samples on the barrel. To detect these barrels the rules are set in a way that also increases the risk of false 


\begin{tabular}{|l||c|c|c||c|c|c|}
\hline \multicolumn{1}{|c||}{} & Barrel & Turret & \\
\hline Targets & True & False & Miss & True & False & Miss \\
\hline $\begin{array}{l}\text { Tanks w barrel } \\
\text { samples }\end{array}$ & 17 & 1 & 1 & 15 & 0 & 3 \\
\hline $\begin{array}{l}\text { Tanks no bar- } \\
\text { rel samples }\end{array}$ & 0 & 0 & 0 & 5 & 0 & 0 \\
\hline APC & 0 & 0 & 0 & 1 & 0 & 0 \\
\hline
\end{tabular}

TABLE II

IDENTIFICATION OF FUNCTIONAL PARTS.

\begin{tabular}{|l|l|l|l|l|l|l|}
\hline \multicolumn{7}{|l|}{ Model } \\
\hline Targets & T72 & M60 & MTLB & BTR70 & M113 & ZSU23 \\
\hline T72 & 9 & 1 & 0 & 0 & 0 & 0 \\
\hline M60 & 0 & 12 & 0 & 0 & 0 & 0 \\
\hline MTLB & 0 & 0 & 4 & 2 & 0 & 0 \\
\hline BTR70 & 0 & 0 & 0 & 2 & 0 & 0 \\
\hline M113 & 0 & 0 & 0 & 0 & 2 & 0 \\
\hline ZSU23 & 0 & 0 & 0 & 0 & 0 & 1 \\
\hline
\end{tabular}

TABLE III

CONFUSION MATRIX BASED ON TARGET TYPES.

detections. In two cases the turret is not detected due to few turret samples, the targets are placed in a forest and trees covers parts of the turret. In one case the turret segment is too far away from the target's inertia, due to outliers on the rear part, to be correctly identified. For all tanks either the barrel or the turret is correctly identified, in most cases both. The ZSU2 has a turret-like construction and it is correctly identified.

\section{E. Matching}

In the information system [3, 4, 15], matching is only performed with models of similar dimensions. To test this approach, all targets are matched with all models. The models $[1,2]$ are described by their 3D structure in low resolution, typically 300-700 frames. If the target's barrel has been identified and the model contains barrel and turret, the model's parts are rotated according to the estimated orientations. Otherwise, the target will be matched with the model in default orientation (barrel pointing straight forward). The target samples are adjusted relative to the model so that their inertia points coincide.

Two confusion matrices are shown for this test case. In Table III the comparisons for the targets with different (target) model types are shown. One T72 is recognized as a M60, that registration contains a quite high noise level. Two MTLB, placed inside a forest and partly occluded, are recognized as BTR70. Further, the T72 and M60 are quite similar in shape and the MTLB and the BTR70 are also quite similar in shape. In Table IV the comparisons for the targets with different (target) model classes are shown. All targets are correctly classified. Matching results for some of the T72 targets are shown in figure 11 .

\section{Discussion AND Future WORK}

The proposed method assumes that most parts of the object have been registered, which demands that the detection method(s) and the target-ground segmentation are stable. This
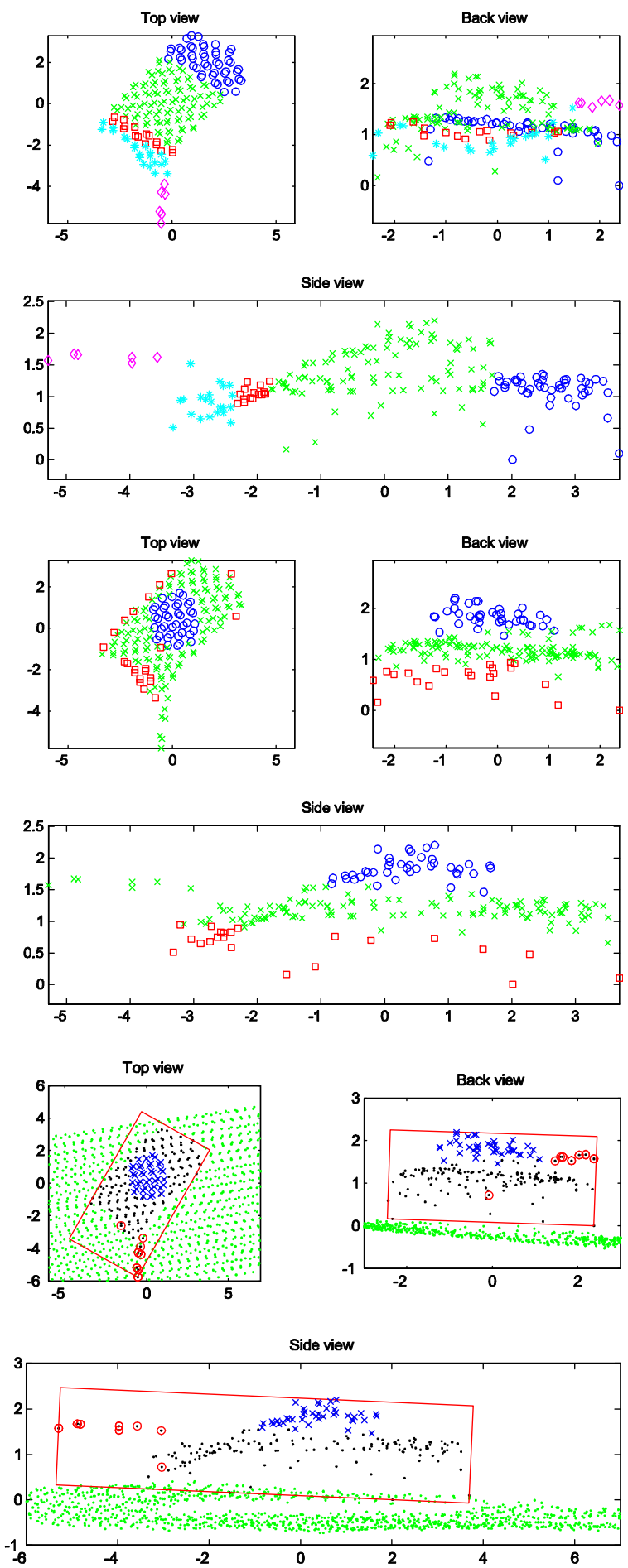

Fig. 8. Result of size and orientation estimation, segmentation and node classification. Top: side view, short side segmentation. Data is divided into five segments, where one is identified as a barrel (marked with rhombus). Middle: side view, long side segmentation. Data is divided into three segments, where one is identified as a turret (marked with circles). Bottom: the rectangles show the estimated size and orientation. Identified barrel samples are marked with 'o' and turret samples with ' $x$ '. Axes in meters. 

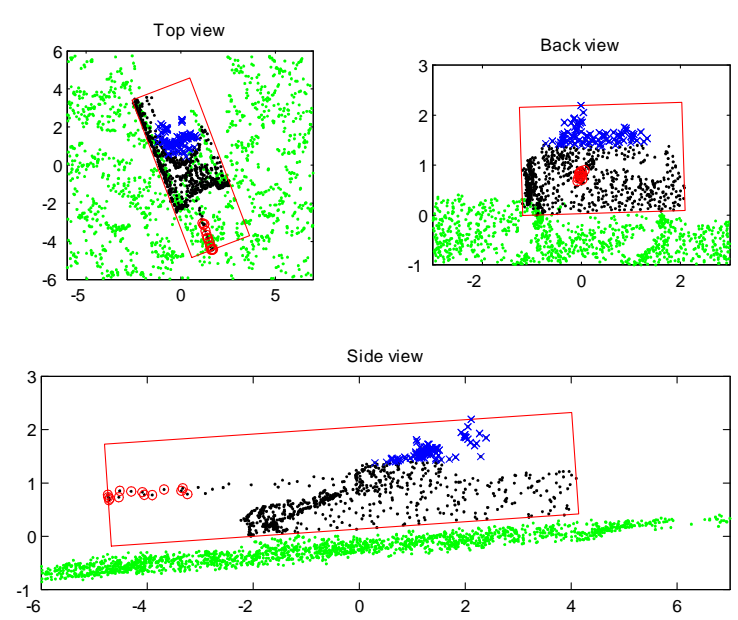

Fig. 9. Result after node classification. The rectangles show the estimated size and orientation. Identified barrel samples are marked with 'o' and turret samples with 'x'. Axes in meters.
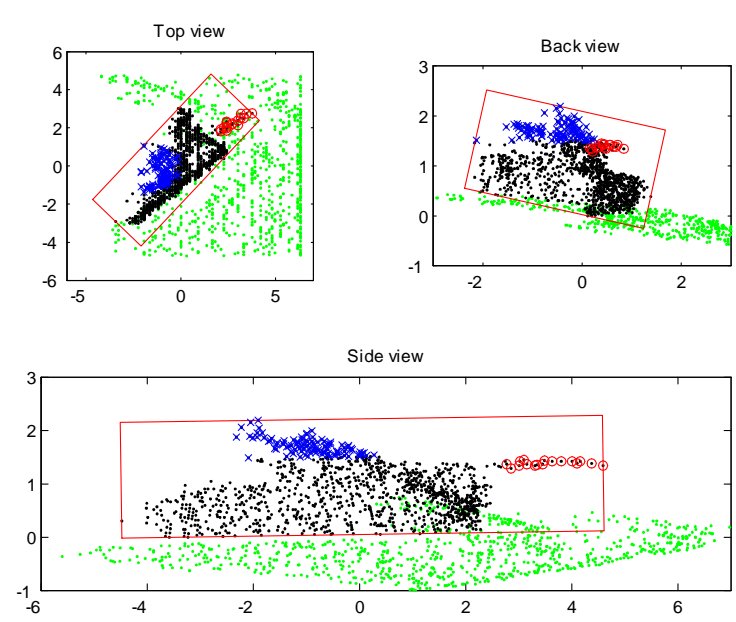

Fig. 10. Result after node classification. The rectangles show the estimated size and orientation. Identified barrel samples are marked with 'o' and turret samples with ' $x$ '. Axes in meters.

is the case when targets are placed in open terrain, but not necessarily in forested terrain. Detection of partly occluded objects needs further research. A laser radar's penetration capability of sparse structures, like vegetation and camouflage nets, is quite large [27,30], which is promising from an ATR perspective. As data is a $3 \mathrm{D}$ scatter, there is some robustness in the method for objects with missing parts.

The rules used for barrel and turret identification in this paper are designed for T72 and M60 tanks. If more tank types are added to the system the rules must probably be adjusted.

We consider data as a 3D point scatter rather than a regular grid (a matrix). The reason for this is that 3D imaging systems may not collect data in matrix format in one single frame but from multiple views. Also, the spatial resolution is often rather low and we may introduce further uncertainties in data by resampling to matrix format.

The rectangle estimation has quite large mean square error

\begin{tabular}{|l|l|l|l|}
\hline & \multicolumn{3}{|l|}{ Model } \\
\hline Targets & Tank & APC & AAG \\
\hline Tank & 22 & 0 & 0 \\
\hline APC & 0 & 10 & 0 \\
\hline AAG & 0 & 0 & 1 \\
\hline
\end{tabular}

TABLE IV

CONFUSION MATRIX BASED ON TARGET CLASSES.
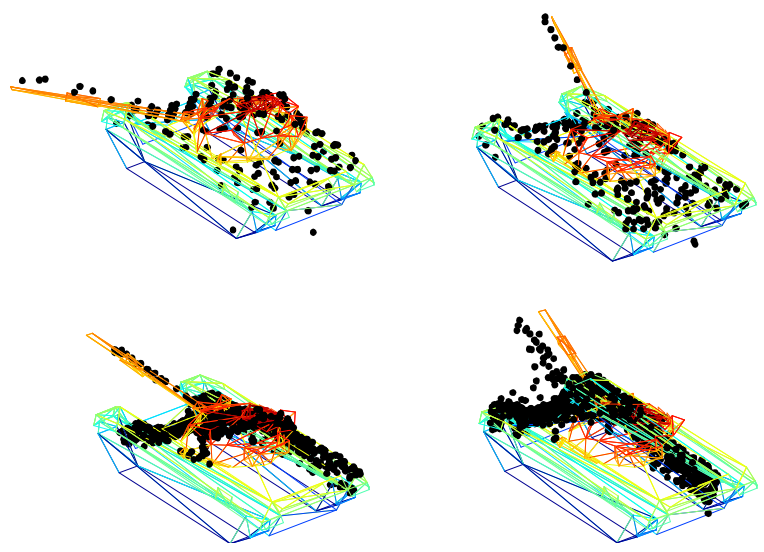

Fig. 11. Matching results, T72 registrations matched with T72 model.

and bias for small samples sets. This means that the estimation error of a small articulated part (like a barrel or door) can be quite large. Further, to obtain good estimates of orientation and dimensions at least two sides of the target must be registered. To take care of these limitations, iterative fitting approaches can be applied in the matching step. Application of an iterative fitting approach can also provide a method that can be used in target identification problems. The intensity values can also be used in this step [33].

The proposed method for 3D size and orientation estimation is fast but not minimum variance. It can be used to get good starting values for more accurate, iterative methods that use both object and surrounding background data [9]. Alternatively, the 3D size and orientation estimates can be used as starting values for more advanced target recognition methods, e.g., [3] and [21].

In the future, we will study detection methods for partly occluded objects. We will also apply iterative approaches in the matching step, to tackle the problems with unsatisfactory initial fits, small data sets and non-consecutive data sets.

\section{CONCLUSIONS}

In this paper an approach to ground target recognition has been proposed. The method is based on general 3D scattered data and can handle arbitrary perspectives of the target. The object recognition algorithm consists of four steps; estimation of the target's 3D size and orientation, target segmentation into parts of approximately rectangular shape, identification of segments that contains the functional parts of the target and finally, matching the target with CAD models.

The core in this approach is rectangle estimation. The proposed rectangle estimation method is minimum variance in orientation estimates but the length and width estimates 
contain bias. The target recognition approach was tested on a small data set of six ground target types, originating from four fundamentally different laser radar systems that operate in three different aspect angles. The width and height estimates are close to the true values, within a three standard deviation limit. The length estimates are underestimated, especially for targets registered in forward-looking perspective. For all tanks either the barrel or the turret was correctly identified, in most cases both. The matching of all targets with all (target) models types resulted in some confusion within the target class. There was no confusion between the three target classes.

The ATR approach is tested on rather few examples, which means that it is hard to draw conclusions on its performance. On the other hand, the approach was tested on data from field experiments from four fundamentally different systems operating in different aspect angles and with different scanning principles. We believe that the approach is promising but further tests are needed.

\section{ACKNOWLEDGMENTS}

The authors appreciate that the data sets were made available and preprocessed. We acknowledge TopEye AB, Pierre Andersson and Tomas Chevalier, FOI Laser Systems.

\section{REFERENCES}

[1] Wire-frame/face 3D Models, http://www.facet3dmodels.com.

[2] Wire-frame/face 3D Models, http://www.3dcadbrowser.com.

[3] J. Ahlberg, L. Klasén, C. Grönwall, M. Ulvklo and E. Jungert, “Automatic target recognition on a multi-sensor platform", in Proc. SSAB, 2003, pp. 93-96.

[4] J. Ahlberg, M. Folkesson, C. Grönwall, T. Horney, E. Jungert, L. Klasén and M. Ulvklo, "Ground Target Recognition in a Query-Based MultiSensor Information System”, Integrated Computer-Aided Engineering Journal, submitted Dec. 2004.

[5] P. Andersson, "Long Range 3D Imaging Using Range Gated Laser Radar Images", Opt. Eng., vol. 45, no. 3, Mar. 2006.

[6] K. S. Arun, T. S. Huang and S. D. Blostein, "Least-squares fitting of two point sets", IEEE Trans. Pattern Anal. Machine Intell., vol. PAMI-9, no 5, pp. 698-700, Sep. 1987.

[7] L. Breiman, J. H. Friedman, R. A. Ohlsen and C. J. Stone, Classification and regression trees, Monterey: Wadsworth and Brooks, 1984, Chapter 8.3.

[8] C. Carlsson, "Vehicle Size and Orientation Estimation Using Geometric Fitting”, Dept. of Electrical Eng., Linköping University, Linköping, Sweden, Jun. 2000, Licentiate Thesis no. 840.

[9] C. Carlsson and M. Millnert, "Vehicle Size and Orientation Estimation using Geometric Fitting", in Proc. SPIE, 2001, vol. 4379, pp.412-423.

[10] C. English, S. Ruel, L. Melo, P. Church and J. Maheux, "Development of a practical 3D automatic target recognition and pose estimation algorithm", in Proc. SPIE, 2004, vol. 5426, pp. 112-123,

[11] H. Freeman and D. Shapira, "Determining the Minimum-Area Encasing Rectangle for an Arbitrary Closed Curve", Communications of the ACM, 1975, vol. 18, no. 7, pp. 409-413.

[12] J. De Geeter, H. Van Brussel, J. De Schutter and M. Decréton, "A smoothly constrained Kalman filter", IEEE Trans. Pattern Anal. Machine Intell., vol. PAMI-19, no. 10, pp. 1171 - 1177, Oct. 1997.

[13] C. Grönwall, T. Carlsson and F. Gustafsson, "Performance analysis of measurement error regression in direct-detection laser radar imaging", in Proc. ICASSP, 2003, vol. VI, pp.545-548

[14] Grönwall, F. Gustafsson and M. Millnert, "Ground target recognition using rectangle estimation", Dept. of Electrical Eng., Linköpings Universitet, Linköping, Sweden, March 2005, Report no.: LiTH-ISY-R-2684.

[15] T. Horney, J. Ahlberg, C. Grönwall, M. Folkesson, K. Silvervarg, J. Fransson, L. Klasén, E. Jungert, F. Lantz and M. Ulvklo M , "An information system for target recognition", in Proc. SPIE, 2004, vol. 5434, pp. 163-175.

[16] D. Huber, A. Kapuria, R. Donamukkala and M. Herbert, "Parts-based 3D object classification", in Proc. CVPR, 2004, vol. 2 , pp. II-82 - II-89.
[17] E. J. Huising and L. M. Gomes Pereira, 'Errors and Accuracy Estimates of Laser Data Acquired by Various Laser Scanning Systems for Topographic Applications", ISPRS Journal of Photogrammetry and Remote Sensing, vol. 53, pp. 245-261, 1998.

[18] B. A. Hutchinson, R. L. Galbraith, B. L. Stann and S. Z. Der, "Simulation based analysis of range and cross-range resolution requirements for the identification of vehicles in ladar imagery", Opt. Eng., vol. 42 , no. 9, pp. 2734-2745, Sep. 2003.

[19] A. V. Jelalian, Laser Radar Systems, Norwood, MA: Artech House, 1992.

[20] A. E. Johnson and M. Hebert, "Using spin images for efficient object recognition in cluttered 3D scenes", IEEE Trans. Pattern Anal. Machine Intell., vol. PAMI-21, no. 5, pp. 433 - 449, May 1999.

[21] L. Klasén, "Image Sequence analysis of Complex Objects. Law Enforcement and Defence Applications", Dept. of Electrical Eng., Linköping University, Linköping, Sweden, Dissertion no. 762, 2002.

[22] E. L. Lehmann and G. Casella, Theory of point estimation. 2nd ed., New York: Springer Verlag, 2001.

[23] H. Pirzadeh, "Computation Geometry with the Rotating Calipers", Faculty of Graduate Studies and Research, McGill University, Canada, Master Thesis, Nov. 1999.

[24] O. Steinvall, H. Olsson, G. Bolander, C. Carlsson and D. Letalick, "Gated viewing for target detection and recognition", in Proc. SPIE, 1999, vol. 3707, pp. 432-448.

[25] O. Steinvall, T. Carlsson, C. Grönwall, H. Larsson, P. Andersson and L. Klasén, "Laser Based 3D imaging. New capabilities for Optical Sensing”, FOI Sensor Technology, FOI, Linköping, Sweden, Tech. Rep. FOI-R-0856-SE, Apr. 2003.

[26] O. K. Steinvall, L. Klasén, C. Grönwall, U. Söderman, S. Ahlberg, Å. Persson, M. Elmqvist, H. Larsson, D. Letalick, P. Andersson, T. Carlsson and M. Henriksson, "3D laser sensing at FOI: overview and a system perspective", in Proc. SPIE, 2004, vol. 5412, pp. 294-309.

[27] O. K. Steinvall, H. Larsson, F. Gustavsson, T. Chevalier, Å. Persson and L. Klasén, "Characterizing targets and backgrounds for 3D laser radar", presented at SPIE Remote Sensing Europe, London, UK, 2004

[28] C. K. Toth, A. Barsi and T. Lovas, "Vehicle recognition from lidar data", in Proc. of ISPRS working group III/3 workshop, 2003, pp. 162-166.

[29] G. Toussaint, "Solving Geometric Problems with the Rotating Calipers", in Proc. IEEE MELECON, 1983.

[30] A. N. Vasile and R. Marino, "Pose-independent automatic target detection and recognition using 3D LADAR data", in Proc. SPIE, 2004, vol. 5426, pp. 67-83.

[31] J. G. Verly and R. L. Delanoy, "Model-based automatic target recognition (ATR) system for forward looking ground based and airborne imaging laser radars (LADAR)", Proc. IEEE., 1996, vol. 84, no. 2, pp. 126-163.

[32] S. Vinson and L. D. Cohen, "Multiple Rectangle Model for Buildings Segmentation and 3D Scene Reconstruction", in Proc. ICPR , 2002, pp. 623-626.

[33] M. R. Wellfare and K. Norris-Zachery, "Characterization of articulated vehicles using ladar seekers", in Proc. SPIE, 1997, vol. 3065, pp. 244254.

[34] T. Yano, T. Tsujimura and K. Yoshida, "Vehicle identification technique using active laser radar system", in Proc. MFI, 2003, pp.275 - 280.

[35] Q. Zheng, S. Z. Der and H. I. Mahmoud., "Model-based Target Recognition in Pulsed Ladar Imagery", IEEE Trans. Image Processing, 2001, vol. 10, no. 4, pp. 565-572, Apr. 2001.

[36] D. Q. Zhu and C.-C. Chu, "Characterization of irregularly shaped bodies",in Proc. SPIE, 1995, vol. 2466, pp. 17-22. 


\section{APPENDIX}

The appendices contain background information and derivations that is not published in the paper. The appendices will be included in the internal report and handed out to the referees.

\section{A. Error distributions of the laser radar systems}

1) General: The measurements are performed in a 3D point scatter $(x, y, z)$. The model for sample $i$ is described by

$$
\begin{aligned}
x_{i} & =x_{i}^{0}+e_{x, i} \\
y_{i} & =y_{i}^{0}+e_{y, i} \\
z_{i} & =z_{i}^{0}+e_{z, i}
\end{aligned}
$$

where $\left(x_{i}^{0}, y_{i}^{0}, z_{i}^{0}\right)$ is the true but unknown coordinate of sample $i$ and $\left(e_{x, i}, e_{y, i}, e_{z . i}\right)$ is the uncertainty in each coordinate. The uncertainties are assumed to be independently distributed in 3D and between samples. Further $\left(e_{x, i}, e_{y, i}, e_{z . i}\right)$ is assumed to have zero mean and variance $\left(\sigma_{e_{x}}^{2}, \sigma_{e_{y}}^{2}, \sigma_{e_{z}}^{2}\right)$, respectively. Calculations of variance in $(x, y, z)$ data gives $\left(X, X^{0}, E_{x}\right.$ etc. are stochastic variables with observations $x_{i}, x_{i}^{0}, e_{x}$ etc.)

$$
\begin{aligned}
& \operatorname{Var}(X)=\operatorname{Var}\left(X^{0}\right)+\operatorname{Var}\left(E_{x}\right)=\sigma_{e_{x}}^{2} \\
& \operatorname{Var}(Y)=\sigma_{e_{y}}^{2} \\
& \operatorname{Var}(Z)=\sigma_{e_{z}}^{2} .
\end{aligned}
$$

In the subsections below the variance in $(x, y, z)$ is derived for three of the data types are used in this paper.

The registered object is rotated an angle $\gamma$ counterclockwise from the $x$ axis. Let $x^{\prime}$ and $y^{\prime}$ describe the main and secondary axis of the object. The relation between $(x, y)$ and $\left(x^{\prime}, y^{\prime}\right)$ is

$$
\left(x^{\prime}, y^{\prime}\right)=(x, y)\left[\begin{array}{cc}
\cos \gamma & \sin \gamma \\
-\sin \gamma & \cos \gamma
\end{array}\right]
$$

The variance in $\left(x^{\prime}, y^{\prime}\right)$ is given by

$$
\begin{aligned}
\operatorname{Var}\left(X^{\prime}\right) & =\operatorname{Var}(\cos \gamma X+\sin \gamma Y) \\
& =\cos ^{2} \gamma \sigma_{e_{x}}^{2}+\sin ^{2} \gamma \sigma_{e_{y}}^{2} \\
\operatorname{Var}\left(Y^{\prime}\right) & =\operatorname{Var}(-\sin \gamma X+\cos \gamma Y) \\
& =\sin ^{2} \gamma \sigma_{e_{x}}^{2}+\cos ^{2} \gamma \sigma_{e_{y}}^{2}
\end{aligned}
$$

and if $\sigma_{e_{y}}^{2}=\sigma_{e_{x}}^{2}$ we have that $\operatorname{Var}\left(X^{\prime}\right)=\operatorname{Var}(X)=\sigma_{e_{x}}^{2}$ and $\operatorname{Var}\left(Y^{\prime}\right)=\operatorname{Var}(Y)=\sigma_{e_{y}}^{2}$.

In the 3D orientation estimation algorithm (Section V-B), the target samples are first studied in $(x, y)$ direction and the orientation $\gamma$ is estimated. Then the target is studied in side view $\left(x^{\prime}, z\right)$ and in back view $\left(y^{\prime}, z\right)$.

2) The TopEye system: The TopEye system is a scanning, down-looking helicopter-carried system. The field tests where the data set was collected is described in Grönwall ${ }^{3}$. The uncertainties in data is described in Huising [17] and also

${ }^{3}$ C. Grönwall, "Mätningar med flygburet multisensorsystem - mätrapport från fordonsplatserna i Kvarn och Tullbron", Dept. of Sensor Technology, Swedish defence research agency (FOI), Linköping, Sweden, Technical Report FOI-D—0060—SE, Aug. 2002 (in swedish). derived in Carlsson ${ }^{4}$. The TopEye company (see Huising) approximates $\sigma_{e_{x}}=\sigma_{e_{y}}=\sigma_{e_{z}}=0.1$ meters. In Carlsson the uncertainties are approximated to $\sigma_{e_{x}}=0.076$ meters, $\sigma_{e_{y}}=0.062$ meters and $\sigma_{e_{z}}=0.072$ meters. The tests of the segmentation that have been performed so far indicates that the segmentation results are similar for both uncertainty approximations. The approximation by the TopEye company are used in this paper.

3) The ILRIS system: The ILRIS system is a hand-carried, scanning laser radar system. The footprint on the target is approximately $0.015-0.02 \mathrm{~m}$ and the distance between samples is approximately $0.3 \mathrm{~m}$ both along and between the scanning lines. The maximum field of view is $40^{\circ} \times 40^{\circ}$ degrees The resulting data, after post processing, is an unordered set of samples $(x, y, z, r)$. The measurement uncertainty is approximately $0.015 \mathrm{~m}$ in $x$ and $y$ and $0.02 \mathrm{~m}$ in $z$ (depth). The laser radar system was placed $5 \mathrm{~m}$ above ground and approximately $190 \mathrm{~m}$ from the target, to constitute forwardlooking perspective.

4) The GV system: The GV data used in this paper originates from early versions of both the measurement system and the generation of $3 \mathrm{D}$ point scatters from range images. The system and the analysis method is described in Andersson ${ }^{5}$.

The analog range data is quantized into $15 \mathrm{~cm}$ range steps (or bins). According to $\mathrm{Taub}^{6}$ this gives a mean square quantization error of $\Delta^{2} / 12$, where $\Delta$ is the step size, thus we have $\sigma_{e_{z}}=0.15 / \sqrt{12}=0.043$ meters. The error in $(x, y)$ is smaller and is after examination of the data set approximated to $\sigma_{e_{x}}=\sigma_{e_{y}}=\frac{1}{2} \sigma_{e_{z}}=0.022$ meters.

\section{B. Properties of the minimum rectangle estimator}

1) Properties of the objective function: The minimization problem to find the rectangle that with minimal area contains the convex hull of the samples is (2):

$$
\min \left(c_{3}-c_{1}\right)\left(c_{4}-c_{2}\right)
$$

subject to

$$
\begin{aligned}
& X_{1, i} \mathbf{n}-c_{1} \geq 0, \quad i=1, \ldots, N \\
& X_{1, i} R \mathbf{n}-c_{2} \geq 0, \quad i=1, \ldots, N \\
& X_{1, i} \mathbf{n}-c_{3} \leq 0, \quad i=1, \ldots, N \\
& X_{1, i} R \mathbf{n}-c_{4} \leq 0, \quad i=1, \ldots, N \\
& \mathbf{n}^{T} \mathbf{n}=1 .
\end{aligned}
$$

Let us study the objective function a bit further. The first four constraints in (2) give that $c_{1}$ and $c_{2}$ will have equal sign and $c_{3}$ and $c_{4}$ will have equal sign. Further, $c_{3}$ and $c_{4}$ will have opposite sign compared with $c_{1}$ and $c_{2}$. This means that if $c_{1}<0, c_{2}<0, c_{3}>0$ and $c_{4}>0$ we have

$\left(c_{3}-c_{1}\right)>0, \quad\left(c_{4}-c_{2}\right)>0 \quad$ and $\left(c_{3}-c_{1}\right)\left(c_{4}-c_{2}\right)>0$.

${ }^{4} \mathrm{C}$. Carlsson, "Calculation of measurement uncertainties in TopEye data", Dept. of Sensor Technology, Swedish defence research agency (FOI), Linköping, Sweden, Technical Report FOA-D-00-00492-408-SE, Jun. 2000.

${ }^{5} \mathrm{P}$. Andersson, "Long-range three-dimensional imaging using range-gated laser radar images", Optical Engineering, vol. 45, no. 03, Mar 2006.

${ }^{6} \mathrm{H}$. Taub and D.L. Schilling, Principles of communication systems, Singapore: McGraw-Hill, 1986, pp.207-209. 
On the other hand, if $c_{1}>0, c_{2}>0, c_{3}<0$ and $c_{4}<0$ we have

$\left(c_{3}-c_{1}\right)<0, \quad\left(c_{4}-c_{2}\right)<0 \quad$ and $\left(c_{3}-c_{1}\right)\left(c_{4}-c_{2}\right)>0$.

This means that the objective function $\left(c_{3}-c_{1}\right)\left(c_{4}-c_{2}\right)$ always will be positive.

2) Properties of the length estimate: The calculations in this section follows $\mathrm{Gut}^{7}$. We have $N$ random samples $X_{1}, X_{2}, \ldots, X_{N}$, that are uniformly distributed, $X \in \mathcal{U}(a, b)$. The unordered samples $X_{i}, i=1, \ldots, N$, have density function $f_{X}(x)=1 /(b-a)$, mean value $E X=(a+b) / 2$ and variance $\operatorname{Var} X=(b-a)^{2} / 12, a \leq x \leq b$. The distribution function is

$$
\begin{aligned}
F_{X}(x) & =\int_{a}^{x} f_{X}(t) d t=\int_{a}^{x} \frac{1}{(b-a)} d t \\
& =\left[\frac{t}{(b-a)}+c\right]_{t=a}^{x} \\
& =\left\{F_{X}(a)=0 \text { gives } c=\frac{-a}{(b-a)}\right\} \\
& =\frac{x-a}{(b-a)}, \quad a \leq x \leq b .
\end{aligned}
$$

We order the samples so that $X_{(1)} \leq X_{(2)} \leq \ldots \leq X_{(N)}$. In a certain orientation $\Phi$ the length $L$ is given by the range of the ordered samples. We first derive the properties for the smallest and the largest samples, i.e., $X_{(1)}$ and $X_{(N)}$, and then go back to the properties of the length estimate.

a) Properties of the smallest sample: The density function of the smallest sample $X_{\min }=X_{(1)}$ is

$$
\begin{aligned}
f_{X_{(1)}}(x) & =N\left(1-F_{X}(x)\right)^{N-1} f_{X}(x) \\
& =N\left(1-\frac{x-a}{(b-a)}\right)^{N-1} \frac{1}{(b-a)} \\
& =\frac{N}{(b-a)^{N}}(b-a-(x-a))^{N-1} \\
& =\frac{N}{(b-a)^{N}}(b-x)^{N-1},
\end{aligned}
$$

${ }^{7}$ A. Gut, An Intermediate Course in Probability, New York: SpringerVerlag, 1995. the expectation value of $X_{(1)}$ is

$$
\begin{aligned}
E X_{(1)} & =\int_{a}^{b} x f_{X_{(1)}}(x) d x \\
& =\frac{N}{(b-a)^{N}} \int_{a}^{b} x(b-x)^{N-1} d x \\
& =\frac{N}{(b-a)^{N}} \\
& =\left[\frac{1}{(-1)^{2}}\left(\frac{(b-x)^{N+1}}{N+1}-\frac{b(b-x)^{N}}{N}\right)\right]_{x=a}^{b} \\
& =-\left(\frac{N}{N+1} \frac{(b-x)^{N+1}}{(b-a)^{N}}-\frac{b(b-x)^{N}}{(b-a)^{N}}\right]_{x=a} \\
& \left.=\frac{(b-a)^{N}}{\left(b-\frac{N}{N+a)^{N}}\right.}\right) \\
& =\frac{b+N a)^{N}}{N+1}(b-a)
\end{aligned}
$$

and the expectation value of $X_{(1)}^{2}$ is

$$
\begin{aligned}
E X_{(1)}^{2}= & \frac{N}{(b-a)^{N}} \int_{a}^{b} x^{2}(b-x)^{N-1} d x \\
= & -\frac{N}{(b-a)^{N}} \\
& {\left[\frac{(b-x)^{N+2}}{N+2}-\frac{2 b(b-x)^{N+1}}{N+1}+\frac{b^{2}(b-x)^{N}}{N}\right]_{x=a}^{b} } \\
= & \frac{N}{(b-a)^{N}} \\
& \left(\frac{(b-a)^{N+2}}{N+2}-\frac{2 b(b-a)^{N+1}}{N+1}+\frac{b^{2}(b-a)^{N}}{N}\right) \\
= & \frac{N^{2} a^{2}+N a^{2}+2 N a b+2 b^{2}}{(N+2)(N+1)} .
\end{aligned}
$$

The variance is

$$
\begin{aligned}
\operatorname{Var} X_{(1)} & =E X_{(1)}^{2}-E^{2} X_{(1)} \\
& =\frac{2 N a b+2 b^{2}+N a^{2}+N^{2} a^{2}}{(N+2)(N+1)}-\left(\frac{b+N a}{N+1}\right)^{2} \\
& =\frac{\left(2 N a b+2 b^{2}+N a^{2}+N^{2} a^{2}\right)(N+1)}{(N+2)(N+1)^{2}} \\
& -\frac{(b+N a)^{2}(N+2)}{(N+2)(N+1)^{2}} \\
= & \frac{N\left(a^{2}-2 a b+b^{2}\right)}{(N+2)(N+1)^{2}} \\
= & \frac{N(b-a)^{2}}{(N+2)(N+1)^{2}} .
\end{aligned}
$$

Examples of mean and variance values are shown in Table V. 


\begin{tabular}{|l|l|l|l|}
\hline$(a, b, N)$ & $E X_{(1)}$ & $E X_{(1)}^{2}$ & $\operatorname{Var} X_{(1)}$ \\
\hline$(-1,1,4)$ & -0.6 & 0.47 & 0.11 \\
\hline$(-1 / 2,1 / 2,4)$ & -0.3 & 0.11 & 0.03 \\
\hline
\end{tabular}

TABLE V

EXAMPLES OF MEAN AND VARIANCE FOR SMALLEST SAMPLES IN X.

\begin{tabular}{|l|l|l|l|}
\hline$(a, b, N)$ & $E X_{(N)}$ & $E X_{(N)}^{2}$ & $\operatorname{Var} X_{(N)}$ \\
\hline$(-1,1,4)$ & 0.6 & 0.47 & 0.11 \\
\hline$(-1 / 2,1 / 2,4)$ & 0.3 & 0.11 & 0.03 \\
\hline
\end{tabular}

TABLE VI

EXAMPLES OF MEAN AND VARIANCE FOR LARGEST SAMPLES IN X. b) Properties of the largest sample: The density function for the largest sample $X_{\max }=X_{(N)}$ is

$$
\begin{aligned}
f_{X_{(N)}}(x) & =N\left(F_{X}(x)\right)^{N-1} f_{X}(x) \\
& =N\left(\frac{x-a}{(b-a)}\right)^{N-1} \frac{1}{(b-a)} \\
& =\frac{N}{(b-a)^{N}}(x-a)^{N-1},
\end{aligned}
$$

the expectation value of $X_{(N)}$ is

$$
\begin{aligned}
E X_{(N)} & =\frac{N}{(b-a)^{N}} \int_{a}^{b} x(x-a)^{N-1} d x \\
& =\frac{N}{(b-a)^{N}}\left[\frac{1}{1^{2}}\left(\frac{(x-a)^{N+1}}{N+1}-\frac{-a(x-a)^{N}}{N}\right)\right] \\
& =\frac{N}{(b-a)^{N}}\left[\frac{(x-a)^{N+1}}{N+1}+\frac{a(x-a)^{N}}{N}\right]_{x=a}{ }^{N} \\
& =\frac{N}{(b-a)^{N}} \frac{(b-a)^{N+1}}{N+1}+\frac{N}{(b-a)^{N}} \frac{a(b-a)^{N}}{N} \\
& =\frac{N}{N+1}(b-a)+a=\frac{N(b-a)+a(N+1)}{N+1} \\
& =\frac{N b+a}{N+1} .
\end{aligned}
$$

and the expectation value of $X_{(N)}^{2}$ is

$$
\begin{aligned}
E X_{(N)}^{2}= & \frac{N}{(b-a)^{N}} \int_{a}^{b} x^{2}(x-a)^{N-1} d x \\
= & \frac{N}{(b-a)^{N}} \\
= & \left.\frac{(x-a)^{N+2}}{N+2}+\frac{2 a(x-a)^{N+1}}{N+1}+\frac{a^{2}(x-a)^{N}}{N}\right]_{x=a}^{b} \\
= & \left.\frac{(b-a)^{N}}{N+2}(b-a)^{2}+\frac{(b-a)^{N+2}}{N+2}+\frac{2 a(b-a)^{N+1}}{N+1}+\frac{a^{2}(b-a)^{N}}{N}\right)_{\text {The }} \\
= & \frac{N(N+1)(b-a)^{2}+N(N+2) 2 a(b-a)}{(N+2)(N+1)} \\
& +\frac{a^{2}(N+2)(N+1)}{(N+2)(N+1)} \\
= & \frac{N^{2} b^{2}+N b^{2}+2 N a b+2 a^{2}}{(N+2)(N+1)}
\end{aligned}
$$

The variance is

$$
\begin{aligned}
\operatorname{Var} X_{(N)} & =E X_{(N)}^{2}-E^{2} X_{(N)} \\
& =\frac{N^{2} b^{2}+N b^{2}+2 N a b+2 a^{2}}{(N+2)(N+1)}-\left(\frac{N b+a}{N+1}\right)^{2} \\
& =\frac{N\left(a^{2}-2 a b+b^{2}\right)}{(N+2)(N+1)^{2}} \\
& =\frac{N(b-a)^{2}}{(N+2)(N+1)^{2}} .
\end{aligned}
$$

Examples of mean and variance values are shown in Table VI.

c) Properties of the length: In a certain orientation $\Phi$ the length $L$ is given by the range of the ordered samples ${ }^{b} X_{(1)} \leq X_{(2)} \leq \ldots \leq X_{(N)}$. I.e.,

$$
L=\operatorname{Range}(X \mid \Phi)=X_{(N)}-X_{(1)} .
$$

The density of length conditioned on the orientation is (Gut, Theorem IV.2.2)

$$
\begin{aligned}
f_{L \mid \Phi}(l)= & N(N-1) \\
& \int_{-\infty}^{\infty}\left(F_{X}(u+l)-F_{X}(u)\right)^{N-2} f_{X}(u+l) f_{X}(u) d u,
\end{aligned}
$$

where $u=x_{(1)}$ and $l=x_{(N)}-x_{(1)}$, which gives $a \leq u \leq b-l$ when $a \leq l \leq b$. The density can now be expressed as

$$
\begin{array}{rl}
f_{L \mid \Phi}(l) & =N(N-1) \\
& \int_{a}^{b-l}\left(\frac{u+l-a}{(b-a)}-\frac{u-a}{(b-a)}\right)^{N-2} \frac{1}{(b-a)^{2}} d u \\
& =\frac{N(N-1)}{(b-a)^{2}} \int_{a}^{b-l}\left(\frac{l}{(b-a)}\right)^{N-2} d u \\
b_{x=a}^{b} & N(N-1) \frac{l^{N-2}}{(b-a)^{N}}[u]_{u=a}^{b-l} \\
= & N(N-1) \frac{l^{N-2}}{(b-a)^{N}}(b-a-l), \quad a \leq l \leq b .
\end{array}
$$

The expectation value is

$$
\begin{aligned}
E(L \mid \Phi) & =E X_{(N)}-E X_{(1)} \\
& =\frac{N b+a-b-N a}{N+1} \\
& =\frac{b(N-1)+a(1-N)}{N+1} \\
& =\frac{b(N-1)-a(N-1)}{N+1} \\
& =\frac{N-1}{N+1}(b-a) .
\end{aligned}
$$




\begin{tabular}{|l|l|l|}
\hline$\left(l_{0}, a, b, N\right)$ & $b(l)$ & $\lg _{10} b(l)$ \\
\hline$(2,-1,1,4)$ & 0.8 & -0.097 \\
\hline$\left(l_{0}, a, b, N\right)$ & $b(l)$ & $\lg _{10} b(l)$ \\
\hline$(1,-1 / 2,1 / 2,4)$ & 0.4 & -0.398 \\
\hline
\end{tabular}

TABLE VII

EXAMPLE OF BIAS IN LENGTH ESTIMATES.

If we set $a=-b$ we have $E(L \mid \Phi)=2 b \frac{N-1}{N+1}$ and $E(L \mid \Phi) \rightarrow 2 b$ as $N \rightarrow \infty$. Thus, this is a biased estimator.

The unconditioned expectation value of $L$ can be derived from

$$
E L=E(E(L \mid \Phi)) \text {. }
$$

For some function $h$ we have

$$
\begin{aligned}
E(E L \mid \Phi) & =E h(\Phi) \\
& =\int_{-\infty}^{\infty} h(\varphi) f_{\Phi}(\varphi) d \varphi \\
& =\int_{-\infty}^{\infty}(E L \mid \Phi=\varphi) f_{\Phi}(\varphi) d \varphi \\
& =\int_{0}^{\pi} \frac{N-1}{N+1}(b-a) \frac{1}{\pi} d \varphi \\
& =\frac{N-1}{N+1} \frac{(b-a)}{\pi}[\varphi]_{\varphi=0}^{\pi} \\
& =\frac{N-1}{N+1}(b-a) .
\end{aligned}
$$

Note that $E L=E(L \mid \Phi)$, which indicates that $L$ and $\Phi$ are independent.

$\mathrm{Kay}^{8}$ defines the bias in the estimates of $L, b(L)$, as

$$
b(L)=E \hat{L}-L_{0},
$$

where $L_{0}$ is the true (but unknown) value and $\hat{L}=$ $\left(\hat{L}_{1}, \hat{L}_{2}, \ldots, \hat{L}_{N}\right)$ are the estimates. Inserting results from previous section, we have

$$
b(L)=\frac{N-1}{N+1}(b-a)-L_{0},
$$

where $(b-a) \approx L_{0}$. We now have

$$
\begin{aligned}
b(L) & =\left(\frac{N-1}{N+1}-1\right) L_{0} \\
& =-\frac{2}{N+1} L_{0},
\end{aligned}
$$

where we can see that $\operatorname{bias}(L) \rightarrow 0$ when $N \rightarrow \infty$. Examples of bias in length and width estimates are shown in Table VII.

Results from Monte Carlo simulation, see Figures 12-13, give that for $N=4$ we have $|b(l)| \approx 10^{-0.1}$. Thus, the simulation agrees with the theory.

In the figures we can also note "knees" in the curves. For low SNR the dominating statistical distribution is the distribution of the noise, i.e., the Gaussian distribution. For high

${ }^{8}$ S. M. Kay, The Fundamentals of statistical signal processing: estimation theory, Upper Saddle River: Prentice Hall, 1993.
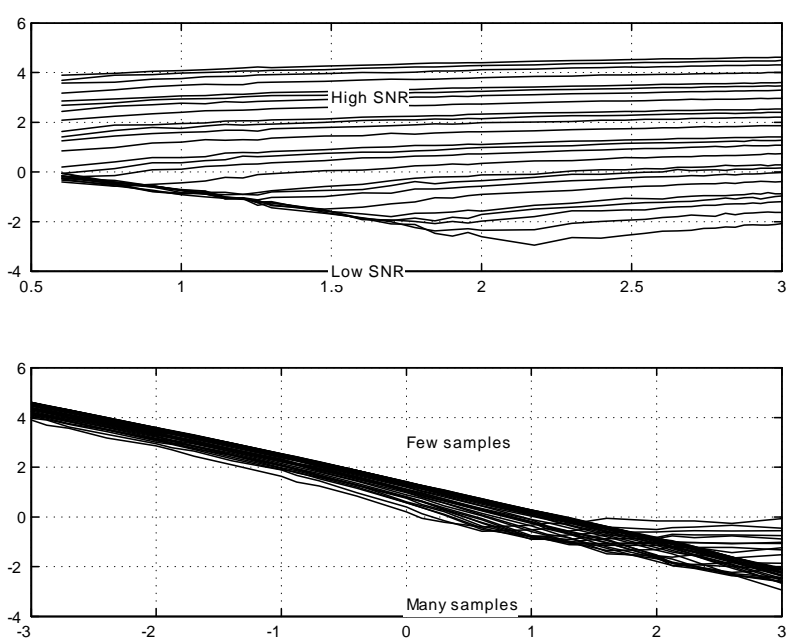

Fig. 12. MSE of length estimate, as a function of number of samples $N$ (upper) and SNR (lower). Logarithmic scale on both axes.
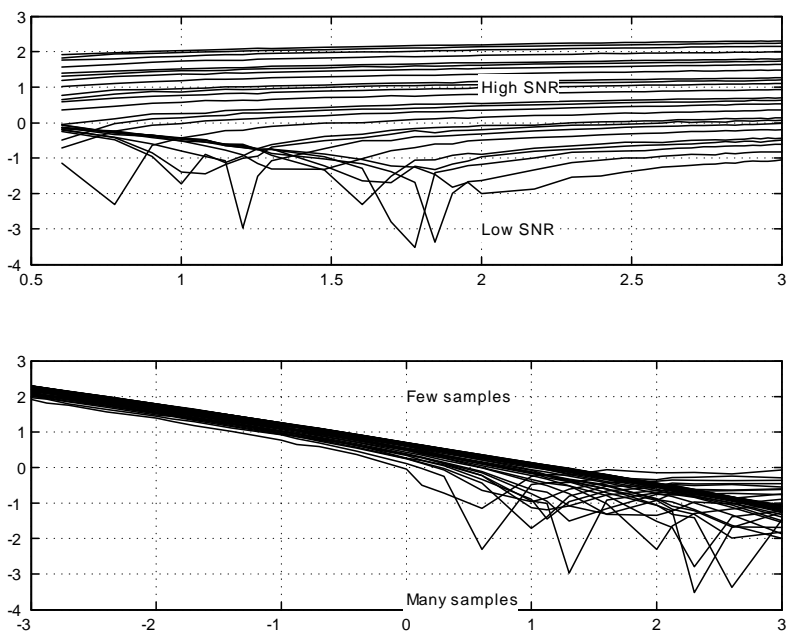

Fig. 13. Bias of length estimate, as a function of number of samples $N$ (upper) and SNR (lower). Logarithmic scale on both axes.

SNR the dominating statistical distribution is the distribution of the samples, i.e., the uniform distribution. We note that for lower SNR more samples are needed to have the uniform distribution as the dominating one. Motivation; For a uniform distribution, the variance is constant both with respect to SNR and number of samples. For Gaussian distribution, the variance is inversely proportional to the number of samples and linearly proportional to the SNR.

3) Properties of the width estimate: We have $N$ random samples $Y_{1}, Y_{2}, \ldots, Y_{N}$, that are uniformly distributed, $Y \in$ $\mathcal{U}(c, d)$. We order the samples so that $Y_{(1)} \leq Y_{(2)} \leq \ldots \leq$ $Y_{(N)}$. In a certain orientation $\Phi+\pi / 2$ the width $W$ is given by the range of the ordered samples. The properties of the smallest and the largest samples, i.e., $Y_{(1)}$ and $Y_{(N)}$, are equal to those of $X_{(1)}$ and $X_{(N)}$ in the previous section. The properties of the width estimates equals those of the length 

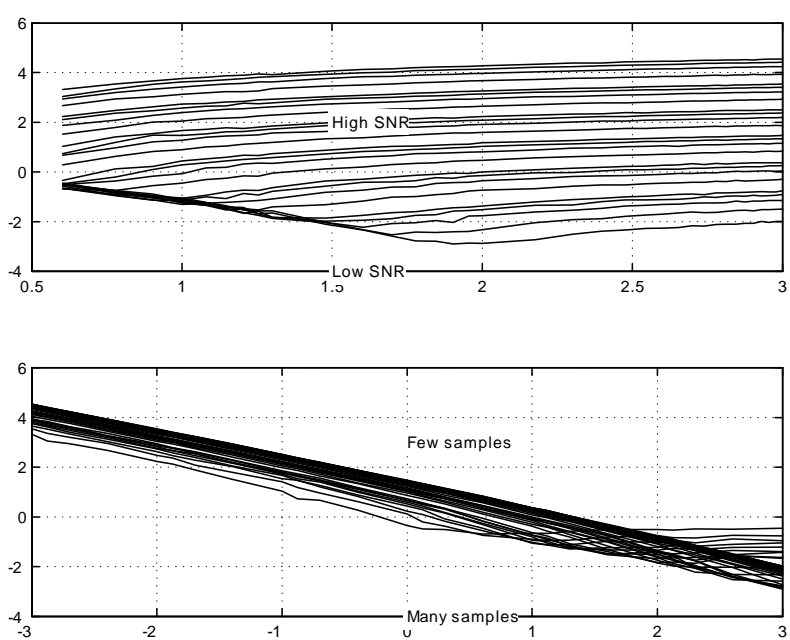

Fig. 14. MSE of width estimate, as a function of number of samples $N$ (upper) and SNR (lower). Logarithmic scale on both axes.
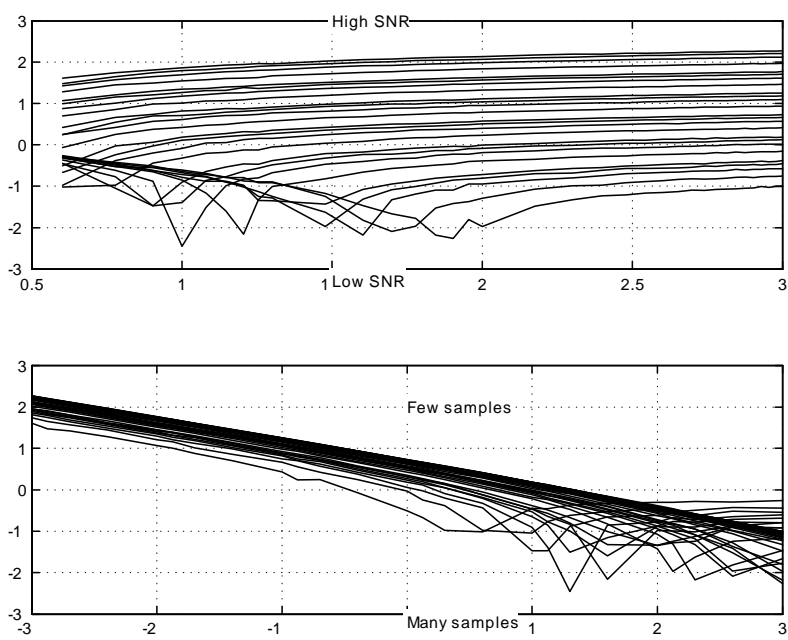

Fig. 15. Bias of width estimate, as a function of number of samples $N$ (upper) and SNR (lower). Logarithmic scale on both axes.

estimate (see previous section). I.e., we have

$$
\begin{aligned}
E(W \mid \Phi)= & \frac{N-1}{N+1}(b-a) \\
& L \text { and } \Phi \text { are independent. } \\
b(W)= & -\frac{2}{N+1} W_{0} .
\end{aligned}
$$

Results from Monte Carlo simulation, see Figures 14-15, give that for $N=4$ we have $|b(w)| \approx 10^{-0.2}-10^{-0.3}$, respectively. Thus, the simulation agrees with the theory (see Table VII).

4) Properties of the orientation estimate: In the Monte Carlo simulations of the orientation estimate $\phi$, the squared bias level is $10-100$ times lower than the mean squared error (MSE). Further, there is no obvious structure in the bias plots. The relation between MSE, bias and estimation variance is

$$
\operatorname{MSE}(\phi)=\operatorname{Var}(\phi)+b^{2}(\phi) .
$$
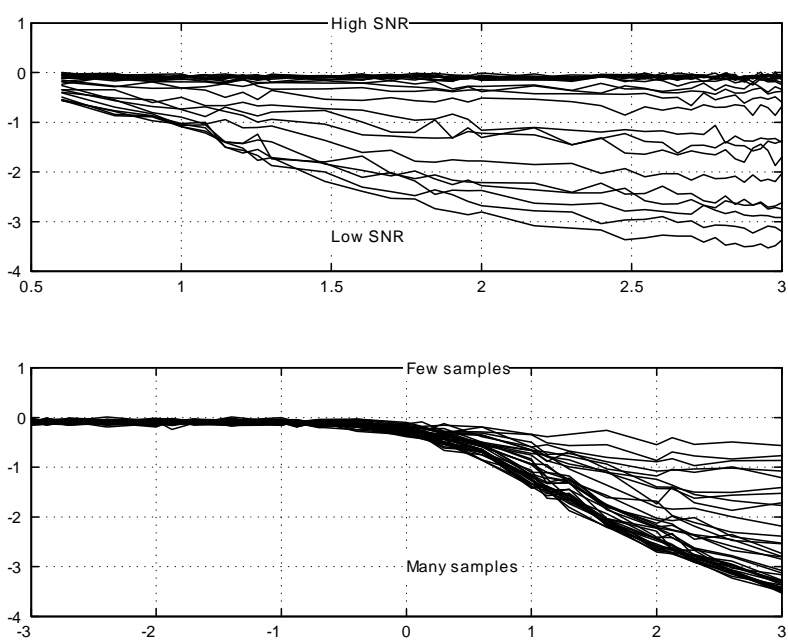

Fig. 16. MSE of orientation estimate, as a function of number of samples $N$ (upper) and SNR (lower). Logarithmic scale on both axes.
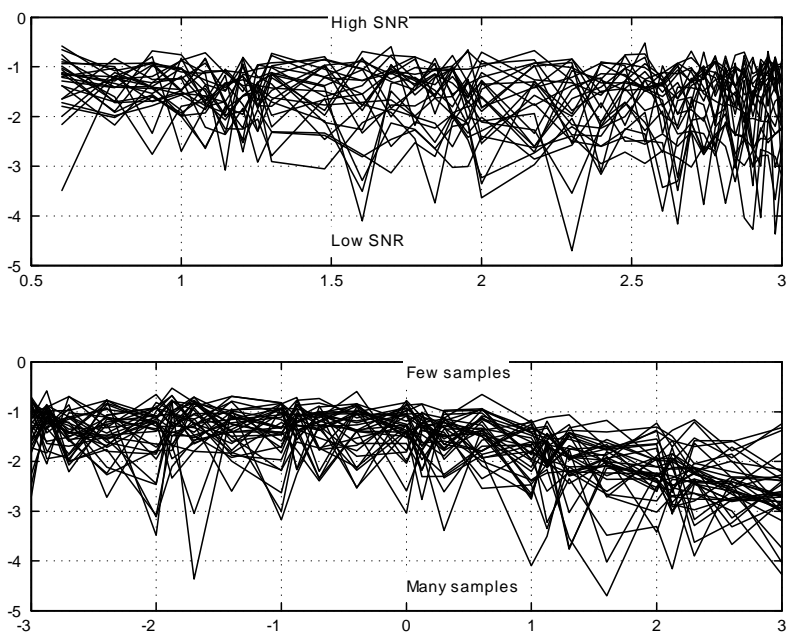

Fig. 17. Bias of orientation estimate, as a function of number of samples $N$ (upper) and SNR (lower). Logarithmic scale on both axes.

In this case $\operatorname{MSE}(\hat{\phi}) \approx \operatorname{Var}(\hat{\phi})$ for all SNR values and all sizes of sample sets.

In the rectangle estimation, the orientation is defined as the angle between the main axis of the rectangle and the $x$ axis. The orientation estimate $\hat{\phi}$ is forced into $[0, \pi]$, as $\phi=\phi+\pi$. Let us assume that $\phi$ uniformly distributed, i.e., $\phi \in \mathcal{U}(0, \pi)$ gives $E \phi=\pi / 2 \approx \pi / 2 \approx 1.571 \approx 10^{0.196}$ and $\operatorname{Var} \phi=$ $\pi^{2} / 12 \approx 0.822 \approx 10^{-0.085}$. In the Monte Carl simulations, $\operatorname{MSE}(\hat{\phi}) \leq 10^{-0.1}$ for all cases. Thus, we can concluded the the orientation estimate is indeed unbiased! Figure 16 and Figure 17 shows the MSE and bias in orientation estimate.

5) Properties of the rectangle area estimate: The rectangle's area, $A_{R}$, is spanned by four points of the convex hull, there is one point on each side of the rectangle. The area is calculated by $A=L W$. In previous section we found that $L$ and $\Phi$ are independent and that $W$ and $\Phi$ are independent. From this, we assume that $L$ and $W$ also are independent. 

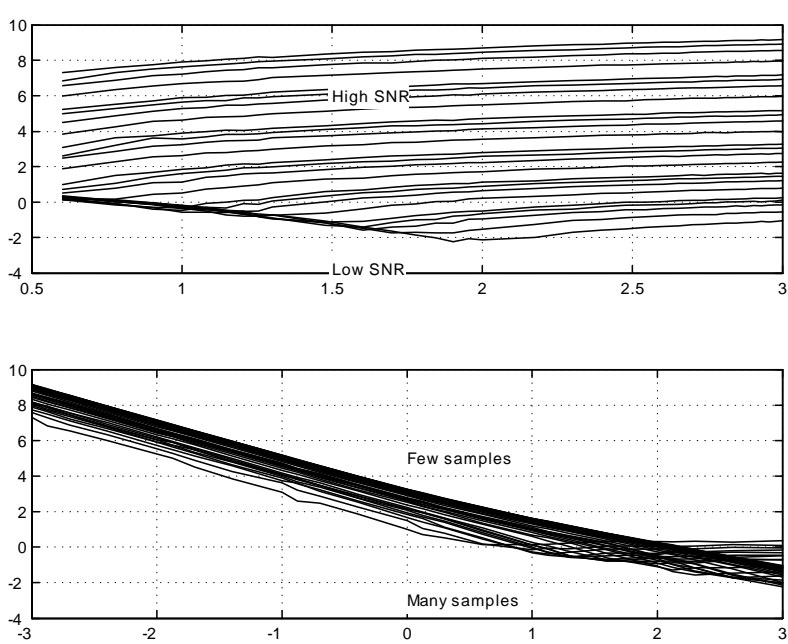

Fig. 18. MSE of area estimate, as a function of number of samples $N$ (upper) and SNR (lower). Logarithmic scale on both axes.

The area's expectation value is then given by

$$
E A=E(L) E(W) \text {. }
$$

The bias in the area estimates can then be expressed as

$$
\begin{aligned}
b(A) & =E(\hat{A})-A_{0} \\
& =E(\hat{L}) E(\hat{W})-A_{0} \\
& =\left(\operatorname{bias}(L)+L_{0}\right)\left(\operatorname{bias}(W)+W_{0}\right)-A_{0} \\
& =\operatorname{bias}(L) \operatorname{bias}(W)+L_{0} \operatorname{bias}(W)+W_{0} \operatorname{bias}(L) \\
& =\frac{4}{(N+1)^{2}} L_{0} W_{0}-\frac{2}{N+1} L_{0} W_{0}-\frac{2}{N+1} L_{0} W_{0} \\
& =\left(\frac{4}{(N+1)^{2}}-\frac{4}{N+1}\right) L_{0} W_{0} \\
& =-\frac{4 N}{(N+1)^{2}} L_{0} W_{0} \\
& =-\frac{4 N}{(N+1)^{2}} A_{0}
\end{aligned}
$$

Example, $l_{0}=2, w_{0}=1$ and $N=4$ gives $|b(A)|=1.28 \approx$ $10^{0.11}$. Results from Monte Carlo simulation gives that for $N=4$ we have $|b(A)| \approx 10^{0.12}-10^{0.18}$, see Figure $18-19$, which is similar to the analytical results.

6) Properties of the area ratio: Where there is low SNR and $N$ is large, the rectangle will approach an ellipse. In that case the area ratio is

$$
\begin{aligned}
\frac{A_{C}}{A_{R}} & =\frac{\pi l w}{4} \frac{1}{l w}=\frac{\pi}{4} \\
& \approx 0.785 \approx 10^{-0.10} .
\end{aligned}
$$

This can be confirmed in the Monte Carlo simulations, see Figure 20.

Ideally, when there are three sample, $N=3$, the convex hull is a triangle and $A_{C} / A_{R}=1 / 2$. When $N$ is small and SNR is either high or low, the bias term is large, i.e., $\operatorname{MSE}\left(A_{C} / A_{R}\right) \approx$ $b^{2}\left(A_{C} / A_{R}\right)$. This is due to that the rectangle estimate is
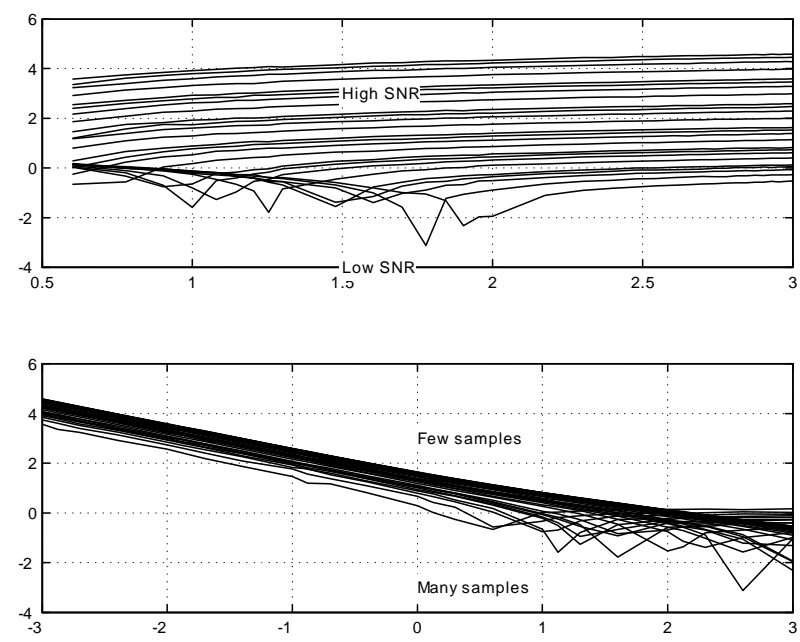

Fig. 19. Bias of area estimate, as a function of number of samples $N$ (upper) and SNR (lower). Logarithmic scale on both axes.

biased (see above). When there are few samples the convex hull will usually not fit the rectangle, thus $A_{C}<A_{R}$ or $A_{C} \ll A_{R}$.

There is not any obvious structure of which samples that are selected for the convex hull, that can be inserted in a sorted statistics framework. We have

$$
\operatorname{bias}\left(\frac{A_{C}}{A_{R}}\right)=E\left(\frac{\hat{A}_{C}}{\hat{A}_{R}}\right)-\left(\frac{A_{C, 0}}{A_{R, 0}}\right) \text {, }
$$

where $A_{C, 0}$ and $E\left(\frac{\hat{A}_{C}}{\hat{A}_{R}}\right)$ are unknown and $A_{R, 0}$ and $E\left(\hat{A}_{R}\right)$ is known. Maybe $E\left(\frac{1}{\hat{A}_{R}}\right)$ can be calculated analytically, but $E\left(\hat{A}_{C}\right)$ is still unknown for the authors.

a) Special case, regular polygon: Let us assume that the segments in the convex hull are of equal length, $a$. In this case, when $n=3$ the convex polygon is a equilateral triangle with area

$$
A_{C}=\frac{\sqrt{3}}{4} a^{2} \approx 0.43 a^{2} .
$$

A polygon with $n$ corners have an area of

$$
A_{C}=\frac{1}{4} n \cot \left(\frac{\pi}{n}\right) a^{2},
$$

where

$$
\begin{aligned}
\cot \left(\frac{\pi}{n}\right) & =\frac{\cos \left(\frac{\pi}{n}\right)}{\sin \left(\frac{\pi}{n}\right)} \approx \frac{1-\frac{1}{2}\left(\frac{\pi}{n}\right)^{2}}{\frac{\pi}{n}-\frac{1}{6}\left(\frac{\pi}{n}\right)^{3}} \\
& =\frac{\frac{1}{2}\left(2-\left(\frac{\pi}{n}\right)^{2}\right)}{\frac{1}{6} \frac{\pi}{n}\left(6-\left(\frac{\pi}{n}\right)^{2}\right)}=\frac{3\left(2-\left(\frac{\pi}{n}\right)^{2}\right)}{\frac{\pi}{n}\left(6-\left(\frac{\pi}{n}\right)^{2}\right)} \\
& \approx \frac{n}{\pi}
\end{aligned}
$$

Let us simplify the rectangle to a square with side $b$ and with area $A_{R}=b^{2}$. For this case the area ratio can be written

$$
\frac{A_{C}}{A_{R}}=\frac{n^{2}}{4 \pi} \frac{a^{2}}{b^{2}} \rightarrow \frac{\pi}{4},
$$



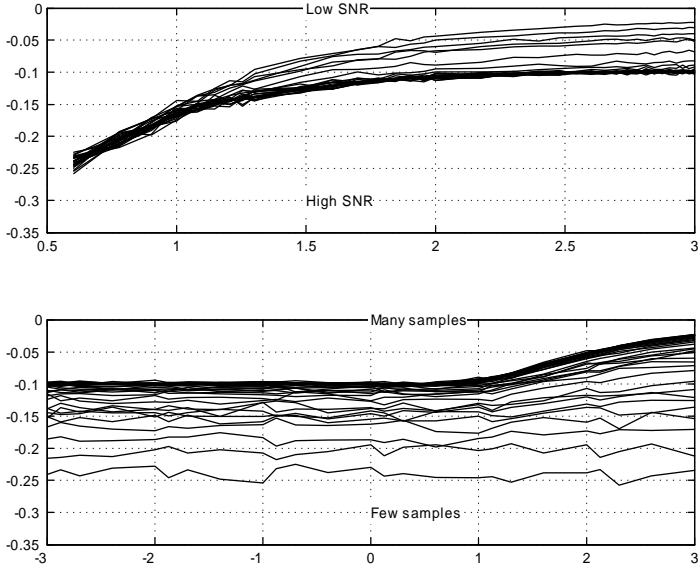

Fig. 20. Mean of quota estimate, as a function of number of samples $N$ (upper) and $S N R$ (lower). Logarithmic scale on both axes.
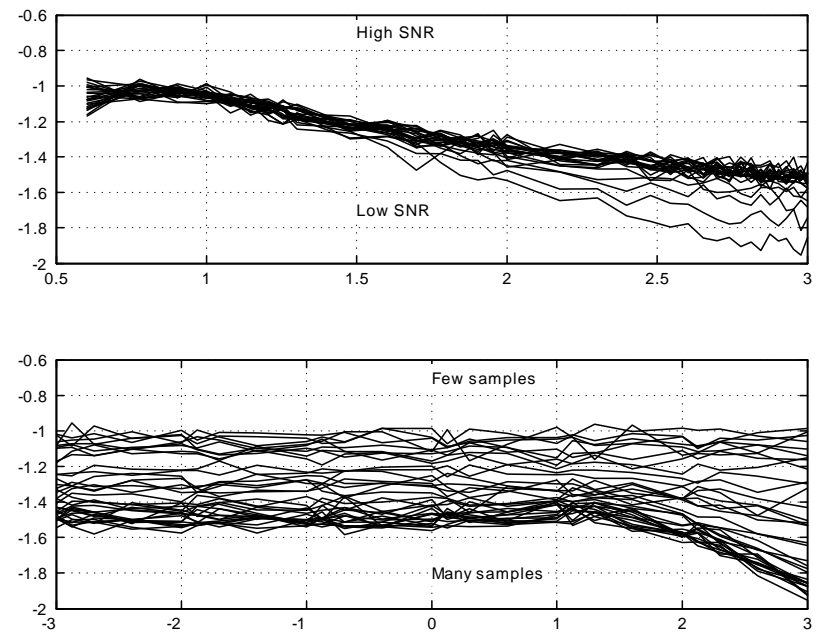

Fig. 21. Standard deviation of quota estimate, as a function of number of samples $N$ (upper) and $S N R$ (lower). Logarithmic scale on both axes.

as the circumference is $n a \approx \pi b$. Mean and variance of the ratio estimate are shown in Figures 20-21. In the Monte Carlo simulations the bias term has a clear structure.

\section{7) Summary:}

- There are clearly bias terms in the length, width and area estimates, but there seems to be no bias in the orientation estimate. Thus, the orientation estimate is minimum variance.

- For small sample sets (high and low SNR) there are not enough samples to describe the rectangle properly and the bias term is dominating. For large sample sets the noise variance is the dominating uncertainty.

- The convex hull approaches a rectangle when the number of samples increases, but how (linear, exponential or logarithmic in $N$ and SNR) is not clear.

- It is hard to predict which points in the convex hull that will be selected for the rectangle estimation. The ideas listed below was fruitless:

- The selected hull points does not have to be the min. and max. points in the 2D domain.

- The selected hull points does not have to be the min. and max. points in polar coordinates.

- The selected hull points does not have to be the points that are furthest away from all other points (calculation performed by dist.m). 


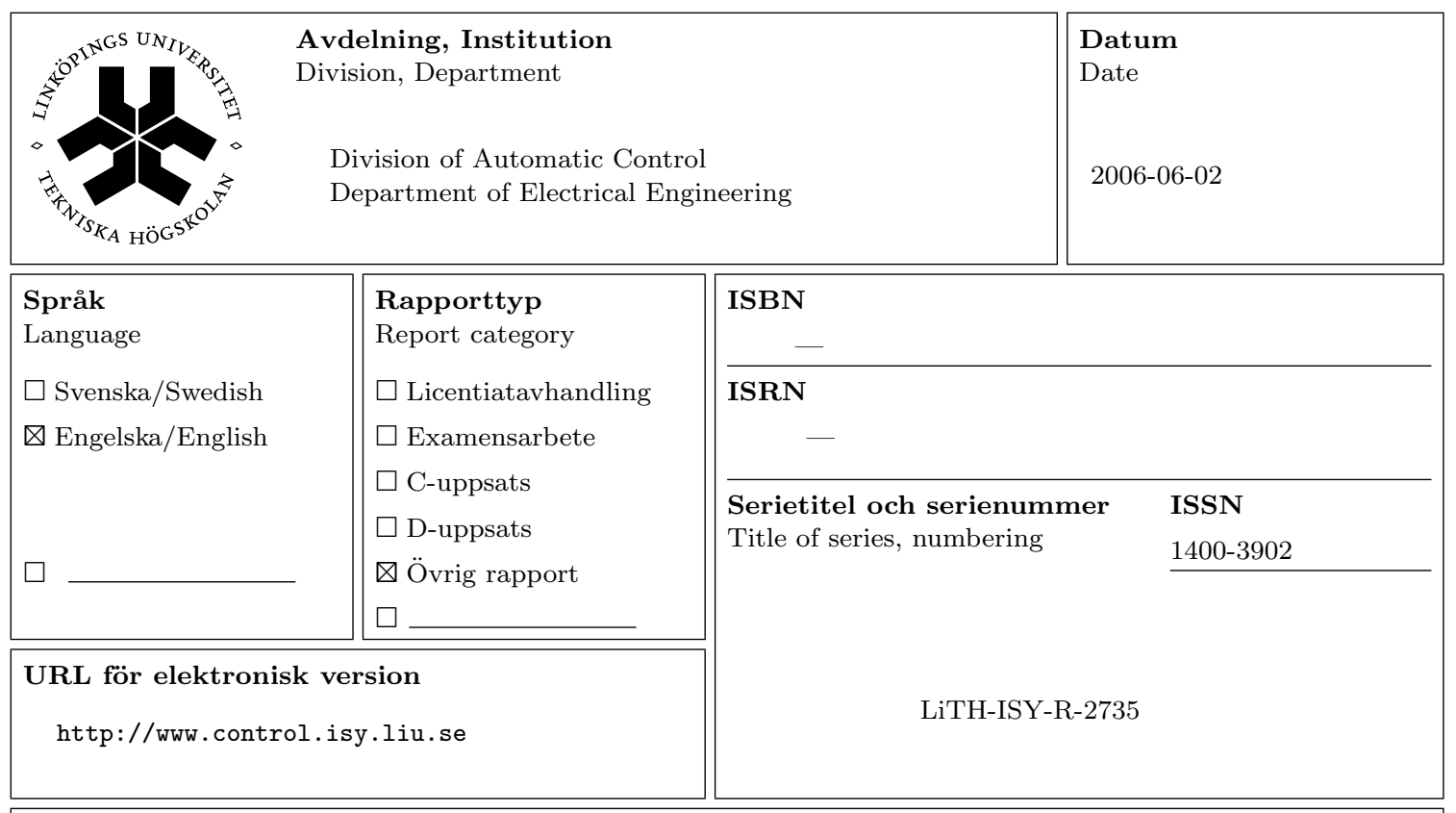

Titel Ground target recognition using rectangle estimation

Title

Ground target recognition using rectangle estination

Författare Christina Grönwall, Fredrik Gustafsson, Mille Millnert

Author

\section{Sammanfattning}

Abstract

We propose a ground target recognition method based on 3D laser radar data. The method handles general 3D scattered data. It is based on the fact that man-made objects of complex shape can be decomposed to a set of rectangles. The ground target recognition method consists of four steps; 3D size and orientation estimation, target segmentation into parts of approximately rectangular shape, identification of segments that represent the targets functional/main parts and target matching with CAD models. The core in this approach is rectangle estimation. The performance of the rectangle estimation method is evaluated statistically using Monte Carlo simulations. A case study on tank recognition is shown, where 3D data from four fundamentally different types of laser radar systems are used. Although the approach is tested on rather few examples, we believe that the approach is promising. 